\title{
NONLINEAR PARAMETRIC EXCITATION OF AN EVOLUTIONARY DYNAMICAL SYSTEM
}

\author{
A Dissertation \\ Presented to the Faculty of the Graduate School \\ of Cornell University \\ in Partial Fulfillment of the Requirements for the Degree of \\ Doctor of Philosophy
}

by

Rocio Esmeralda Ruelas

January 2015 
(C) 2015 Rocio Esmeralda Ruelas

\section{ALL RIGHTS RESERVED}




\section{NONLINEAR PARAMETRIC EXCITATION OF AN EVOLUTIONARY DYNAMICAL SYSTEM Rocio Esmeralda Ruelas, Ph.D.}

Cornell University 2015

Evolutionary Dynamics is a field that combines Dynamical Systems with Game Theory. Game Theory studies the costs and benefits of competing strategies. This competition between strategies is called a "game" and is usually represented by a payoff matrix. The entries of the payoff matrix represent the loss or gain received when one type of strategy plays against another. Through the use of differential equations known as the replicator equations, we can use the information in the payoff matrix to model the change in relative population (or frequency) for all of the strategies. Once we have written the evolutionary equations we can study the dynamics using the methods and theorems developed in the field of Dynamical Systems.

The Rock-Paper-Scissor model is used to describe systems where there are three strategies and where each strategy has an advantage over one strategy, but a disadvantage over the other strategy. The model is named after the classic game in which Rock beats Scissors, Scissors beats Paper, and Paper beats Rock. Using the replicator equations, we can model the changes that occur in the relative populations of the strategies. Strategies whose payoffs are relatively better will have increasing population frequencies while those with lower payoffs will have decreasing population frequencies.

In this work, we consider a variation of the standard RPS game where the payoffs vary periodically in time. In particular, we consider a model with the 
following payoff matrix.

$$
\begin{gathered}
R \\
P \\
P
\end{gathered}\left(\begin{array}{ccc}
R & S \\
0 & -1+A_{1} \cos \omega t & 1+A_{2} \cos \omega t \\
1+A_{3} \cos \omega t & 0 & -1+A_{4} \cos \omega t \\
-1+A_{5} \cos \omega t & 1+A_{6} \cos \omega t & 0
\end{array}\right)
$$

We began our investigation by considering a simple case of our model where we set $A_{1}=-A_{2}=A$ and $A_{3}=A_{4}=A_{5}=A_{6}=0$ thus reducing the number of parameters down to two. For these parameters we found that, generally, the solutions to the associated replicator equations were quasiperiodic. For some values of $A$ and $\omega$, solutions that started near the interior equilibrium point would initially move away from the equilibrium point before eventually returning. Using a linear perturbation method, we were able to determine the parameter regions for which this behavior occurred. These parameter regions resemble the tongues of instability characteristic of Mathieu's equation. We were also able to determine the effects of nonlinear terms by deriving and analyzing equations for the slow flow of the replicator equations. We compared those results to numerically generated Poincaré maps and found that they agreed for small perturbations.

Next we considered a subset of parameters in our proposed payoff matrix (1) where the interior equilibrium point persists. This results in the following conditions,

$$
\begin{aligned}
& A_{1}=A_{6}+A_{5}-A_{2} \\
& A_{3}=A_{6}+A_{5}-A_{4}
\end{aligned}
$$

We used subharmonic resonance to locate the regions in parameters space where the interior equilibrium point exhibited linear resonance. We were sur- 
prised to discover that for a subset of parameters these regions of linear resonance disappeared according to numerical approximations. We then proved analytically that they did in fact disappear. Finally, we extended our analytical proof so that it applied to a family of two-dimensional dynamical systems with time-varying periodic terms. 


\section{BIOGRAPHICAL SKETCH}

Rocio E. Ruelas was born on September 17, 1986 in Jalisco, Mexico. At the age of four, she moved with her family to California where she began kindergarten. Although she did not know any English, Rocio enjoyed school and quickly caught up to the rest of her classmates. In high school, Rocio excelled in mathematics, but loved learning about the applications in her physics class. Thus, she decided to pursue a physics major in college.

Rocio attended Harvey Mudd College where she received a B.S. in Physics in 2008. During her time there, she participated in a number of events concerning diversity in STEM fields and also volunteered as a tour guide. She had the opportunity to conduct astrophysics research with Professor Ann Esin, but realized through the experience that did not want to become an astrophysicist. The following summer, she conducted research on network clusters at the University of Michigan - Ann Arbor with Professor Michael Bretz. She very much enjoyed the mathematical and computational aspects of her research. Hence, when Rocio applied to graduate schools, she chose programs in Applied Mathematics and Mathematical Physics.

After college, Rocio attended the Applied Mathematics program at Cornell University and received a Cornell Sloan Fellowship for her first three years. She immediately started working with Professor Richard Rand and managed to publish five papers during her time there. Rocio continued to participate in diversity events at Cornell University and volunteered as a Registration Chair for the Cornell's Expanding Your Horizons workshop. After her thesis defense, Rocio plans to teach mathematics courses at Moreno Valley College. 
To the Center for Applied Mathematics: Thanks for the memories! 


\section{ACKNOWLEDGEMENTS}

I have been blessed to be surrounded by a number of people who have supported me and believed in me throughout my graduate studies. I am forever grateful to them and hope to one day repay their kindness.

First and foremost, I would like to thank my advisor Richard Rand for his patience, guidance, and understanding. Aside from teaching me a great deal of Nonlinear Dynamics, Professor Rand has been an incredible example of what it means to be a great mentor, researcher, educator, and friend. It is suffice to say that this thesis would not have been completed without him. I would also like to thank the members of my committee, Steve Strogatz and Tim Healey, for their encouragement and direction.

My time in Ithaca might not have been as wonderful without the efforts of Sara Xayarath Hernandez and the Diversity Programs in Engineering. It was inspiring as well as incredibly comforting to be surrounded by people passionate about diversity issues. Thank you for all the support, not to mention the free lunches.

I would like to thank the Alfred P. Sloan foundation and Cornell University for their financial support in the form of graduate fellowships. I feel very fortunate to have had the opportunity to pursue my research without having to worry about funding.

Finally, I would like to thank my friends and family for keeping me smiling and grounded. I am especially grateful to my parents for their encouragement and sacrifice throughout the years. 


\section{TABLE OF CONTENTS}

Biographical Sketch . . . . . . . . . . . . . . . iii

Dedication ..................... iv

Acknowledgements . . . . . . . . . . . . . . v v

Table of Contents . . . . . . . . . . . . . . . vi

List of Figures $\ldots \ldots \ldots \ldots \ldots \ldots \ldots \ldots \ldots$ vii

1 Synopsis 1

1.1 Motivation . . . . . . . . . . . . . . . 1

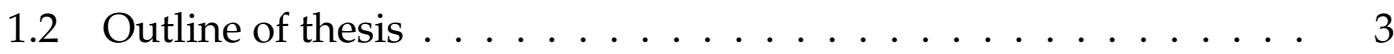

2 Introduction into Evolutionary Games 5

2.1 Matrix Games and Nash Equilibria . . . . . . . . . . . . . 5

2.2 Evolutionary Stable Strategies . . . . . . . . . . . . . . . . 6

2.3 Replicator Equations . . . . . . . . . . . . . . . . 8

3 Rock-Paper-Scissor Model 11

3.1 Dynamics of Standard RPS Model . . . . . . . . . . . . . . 12

3.2 Applications of RPS models . . . . . . . . . . . . . . . . . . 15

4 RPS Model with Periodic Time-Varying Payoffs - A Simple Case 19

4.1 Model . . . . . . . . . . . . . . . . . . . . . . . . . 19

4.2 Simple Case . . . . . . . . . . . . . . . . . . . . 20

4.3 Linear Resonance . . . . . . . . . . . . . . . . . . . . . . . 21

4.4 Multiple Scales Perturbation Method . . . . . . . . . . . . . . . 24

4.5 Poincaré map . . . . . . . . . . . . . . . . . . 27

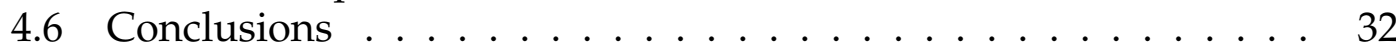

5 RPS Model with Periodic Time-Varying Payoffs - The General Case 36

5.1 Subharmonic Resonance . . . . . . . . . . . . . . . . . . . . 37

5.2 Disappearing Tongue . . . . . . . . . . . . . . . . . . 42

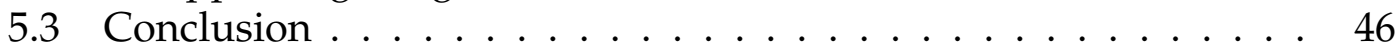

6 Disapperance of Resonace Tongues 48

6.1 A Theorem . . . . . . . . . . . . . . . . . 52

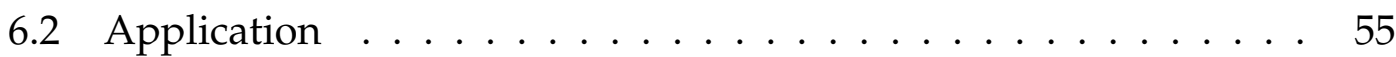

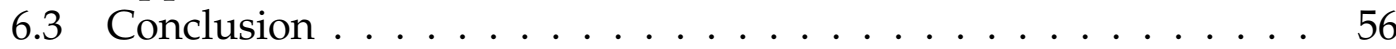

$\begin{array}{lll}7 & \text { Conclusion } & 58\end{array}$

$\begin{array}{ll}\text { Bibliography } & 60\end{array}$ 


\section{LIST OF FIGURES}

3.1 Solutions to the standard RPS model plotted in two dimensions. The values of $x_{3}$ can be found by using $x_{3}=1-x_{1}-x_{2}$. As expected, there is an equilibrium at $x_{1}=x_{2}=x_{3}=\frac{1}{3}$ and it is a center surrounded by neutral oscillations. . . . . . . . . .

4.1 Motions of eqs. (4.5), (4.6) for $A=0.02$ and $\omega=1$ obtained by numerical integration. Here the periodic motions of figure 3.1 are replaced by quasiperiodic motions. Note that motions starting near the equilibrium point $\left(\frac{1}{3}, \frac{1}{3}\right)$ remain near it. . . . . . . . .

4.2 Motion of eqs. (4.5), (4.6) for $A=0.1$ and $\omega=1.154$ for initial conditions $x_{1}=x_{2}=0.333$ obtained by numerical integration. Note that here a motion which starts near the equilibrium $\left(\frac{1}{3}, \frac{1}{3}\right)$ travels far away from it. . . . . . . . . . . . . . . . . .

4.3 Bifurcation curves (4.41) showing the number of nontrivial equilibria in the slow flow (4.36),(4.37). See figure 4.4 for sample phase portraits of the slow flow in each of these regions. . . . . .

4.4 Plot of the first integral (4.42) for various values of $k$. Note, that as $k$ decreases, the system traverses the tongue in figure 4.3 from right to left and the number of nontrivial equilibria changes. . . .

4.5 Poincaré map obtained by numerically integrating eq.(4.21) for $\epsilon=0.1$ and $\omega=2 / \sqrt{3}+k \epsilon^{2}$. Cf. figure 4.4. . . . . . . . . .

4.6 Poincaré map obtained by numerically integrating eq.(4.21) for $\epsilon=1$ and $\omega=2 / \sqrt{3}+k \epsilon^{2}$. Cf. figure 4.5. . . . . . . . . .

4.7 Motion of eqs. (4.5), (4.6) for $A=0.1$ and $\omega=\frac{2}{\sqrt{3}}-\left(\frac{1}{3 \sqrt{3}}+0.2\right) A=$ 1.1155 for initial conditions $x_{1}=x_{2}=0.3$ obtained by numerical integration. This system lies to the left of the resonance tongue in figure 4.3. Note that here a motion which starts near the equilibrium $\left(\frac{1}{3}, \frac{1}{3}\right)$ travels far away from it. . . . . . . . . . . . .

4.8 Motion of eqs. (4.5), (4.6) for $A=0.1$ and $\omega=\frac{2}{\sqrt{3}}+\left(\frac{1}{3 \sqrt{3}}+0.2\right) A=$ 1.1939 for initial conditions $x_{1}=x_{2}=0.3$ obtained by numerical integration. This system lies to the right of the resonance tongue in figure 4.3. Note that here a motion which starts near the equilibrium $\left(\frac{1}{3}, \frac{1}{3}\right)$ remains close to it. . . . . . . . . . .

5.1 2:1 subharmonic resonance tongue, eq.(5.43). The RPS equilibrium point at $x_{1}=x_{2}=\frac{1}{3}$ is linearly unstable for parameters inside the tongue. The presence of nonlinearities detunes the resonance and prevents unbounded motions which are predicted by the linear stability analysis. . . . . . . . . . . . . . . . 
6.1 The boundaries of the resonance tongue calculated numerically. Red is when $\mu=0.5$, black is when $\mu=0.7$, and blue is when $\mu=0.9$. The boundaries of the tongue intersect multiple times and more frequently as $\mu \rightarrow 1 . \ldots \ldots \ldots \ldots$. . . . . 51 
CHAPTER 1

\section{SYNOPSIS}

The field of Evolutionary Dynamics has grown tremendously since its conception in the 1940's and continues to be an exciting area of research. While many of the principles of evolution have long been accepted as fact in the scientific community, there still remain a number of unanswered questions regarding the emergence of certain behaviors or characteristics. The most famous example being the prominence of cooperation in a variety of species including humans. If individuals or genes are said to act in their own best interest why then do they cooperate? Evolutionary Dynamics gives us the tools to address these types of questions by describing behaviors as strategies and assigning benefits and costs

to each strategy. Furthermore, the population frequency of those strategies can be described through differential equations where the population frequency of strategies rise and fall based on their relative fitness. By studying the dynamics of these differential equations we can determine under what conditions certain strategies become prevalent in the species. In the case of cooperation, it has been shown that repeated interactions can lead to the success of a tit-for-tat strategy which cooperates unless the opposing player has previously defected [2].

\subsection{Motivation}

In this work, we focus our attention on a specific type of Evolutionary Game known as Rock-Paper-Scissors (RPS). Like the children's game with the same name, the RPS game consists of three strategies where Rock has an advantage over Scissors which has an advantage over Paper which in turn has an advan- 
tage over Rock. The version of the RPS game where all strategies have equal benefits and costs is known as the standard RPS model. The population dynamics associated with this model result in cycles where the highest population frequency alternates between Rock, Paper, and Scissors [6, 7]. In general, all three strategies will remain in some proportion in the population. This behavior makes the RPS model a useful tool in understanding how biodiversity can exists in biological or sociological systems.

In this work, we wish to expand on a RPS model originally proposed by Rand et al. [15] where time-varying periodic terms are added to the standard RPS payoff matrix. These terms could potentially be used to model seasonal variation in biological systems. While the model proposed by Rand et al. [15] only added time-varying periodic terms to two entries in the payoff matrix, we extended the model to include periodic terms in all the non-zero entries of the standard RPS payoff matrix. Thus, our model has the following form:

$$
R\left(\begin{array}{ccc}
R & P & S \\
0 & -1+A_{1} \cos \omega t & 1+A_{2} \cos \omega t \\
1+A_{3} \cos \omega t & 0 & -1+A_{4} \cos \omega t \\
-1+A_{5} \cos \omega t & 1+A_{6} \cos \omega t & 0
\end{array}\right)
$$

We then analyzed the expanded model by using methods from Nonlinear Dynamics. 


\subsection{Outline of thesis}

We begin this work by providing an introduction to evolutionary games in Chapter 2. A brief history of the field is given and notable terms such as Nash equilibrium, evolutionary stable strategy, and payoff matrix are defined and explained. We further describe the use of replicator equations to model population dynamics of systems with an underlying corresponding payoff matrix.

In Chapter 3 we formally introduce Rock-Paper-Scissor (RPS) models and discuss the dynamics of the canonical RPS model known as the standard model. We identify the equilibrium points and general behavior of the standard RPS model. Applications and prominent papers relating to RPS models are also discussed.

Chapter 4 introduces the RPS model with time varying coefficients that is the focus of this work. We consider a simple case with only two parameters and use perturbation methods to find the regions in parameter space where the interior equilibrium exhibits linear resonance. Next, we study the system including nonlinear terms by using a more powerful perturbation method called multiple scales. This results in slow flow equations which reveal bifurcation in the structure of periodic motions in the neighborhood of linear resonance. The

results are then compared with numerically generated Poincaré maps for the full nonlinear system.

Chapter 5 delves further into the time varying RPS model and investigates the dynamics of the model under the condition that the interior equilibrium point persists. Using subharmonic resonance, we are able to determine the boundaries in parameter space that separate the regions of instability of the inte- 
rior equilibrium from those of stability. These regions are referred to as tongues of instability. Numerical results suggest that there exists combinations of parameters for which the tongues of instability disappear. But numerical and perturbation results are approximate, whereas the question of whether or not the tongue disappears is exact. Thus, we proved a theorem to show that the tongues actually do close up.

In Chapter 6 we generalize the disappearing tongue theorem of Chapter 5 to apply to a class of linear, periodically forced, dynamical systems.

We conclude our findings in Chapter 7 and discuss the implications of our results. 
CHAPTER 2

INTRODUCTION INTO EVOLUTIONARY GAMES

\subsection{Matrix Games and Nash Equilibria}

In 1944, John von Neumann wrote the groundbreaking text, Theory of Games and Economic Behavior, in which the field of Game Theory was born [23]. The book has inspired a mountain of interdisciplinary research, most of which focuses on two-player matrix games. In two-player matrix games, we imagine that there is a set of strategies $S$ from which a player may choose. For each game played we assume that each player chooses a strategy without having any knowledge of what strategy the opposing player chooses. The choices are then revealed and the payoffs, i.e. the costs and benefits, are given. All possible outcomes to the game can be represented in a matrix where the rows of the matrix correspond to the strategy chosen by the first player and the columns correspond to the strategy of the second player.

Let us consider a simple example where we only have two strategies $(A$ and $B)$. The most general form of the payoff matrix would look like,

$$
\begin{aligned}
& \text { A B } \\
& A\left(\begin{array}{ll}
a_{11}, b_{11} & a_{12}, b_{21} \\
a_{21}, b_{12} & a_{22}, b_{22}
\end{array}\right)
\end{aligned}
$$

where the values $a_{i j}$ denote the payoff that Player 1 receives when it plays the strategy in row $i$ and Player 2 plays the strategy in column $j$. Similarly, $b_{i j}$ is the payoff that Player 2 receives when it plays the strategy in column $i$ and Player 1

plays the strategy in row $j$. In this work, we assume that the game is symmetric, 
meaning that there are no differences between players and $a_{i j}=b_{j i}$. In this case the payoff matrix can be simplified to the following,

$$
\begin{gathered}
A \\
A \\
B\left(\begin{array}{cc}
a_{11} & a_{12}, \\
a_{21} & a_{22},
\end{array}\right)
\end{gathered}
$$

One of the major questions that is asked when analyzing games is what strategies will rational players choose. This question lead to the development of one of the most significant notions in game theory, the Nash equilibrium. Intuitively, strategies that are Nash equilibria are the best response to themselves. Meaning that if each player was playing a Nash equilibria, no player can increase their payoff by switching strategies. More formally, the Nash equilibrium is defined as,

$$
p \cdot U q \leq q \cdot U q
$$

where $U$ is the payoff matrix, $q$ is the Nash equilibrium, and $p$ is any other strategy. Thus, $p \cdot U q$ is the payoff a player receives when playing strategy $p$ against strategy $q$. Then $q$ is a Nash equilibrium when no other strategy $p$ does better against than it does against itself.

\subsection{Evolutionary Stable Strategies}

While the Nash equilibrium is a very important concept in game theory, it is not able to explain how certain strategies have become prevalent in a population. 
This limitation was explored in a paper by Smith and Price titled "The Logic of Animal Conflict."[19] Smith and Price sought to understand why animals seem to end conflicts before either animal is seriously harmed. They devised a twoplayer game designed to mimic animal conflict. In a altercation, an animal may choose to use conventional tactics (C), which are unlikely to to cause serious injury, or dangerous tactics (D) which are likely to cause serious harm. This game is now more famously known as the Hawk-Dove game or CooperationDefection game. An example payoff matrix for this type of game would be,

$$
\begin{aligned}
& \text { C D } \\
& \text { C }\left(\begin{array}{ll}
2 & -2 \\
3 & -1
\end{array}\right)
\end{aligned}
$$

From (2.3) we see that if all the animals use conventional tactics they all benefit, but if just a few use dangerous tactics then those individuals will receive a higher payoff. Therefore, no matter what the opponent does it is always best to use dangerous tactics and D is a Nash equilibrium. However, in nature, we observe that animals tend to use conventional rather than dangerous tactics. For example, in many snake species the males fight each other by wrestling without using their fangs [19]. How can the prevalence of conventional tactics be explained?

Smith and Price were able to explain the rise of conventional tactics by changing their model to include repeated interactions and mixed strategies. By including repeated interactions, the animal's choice can now be informed by the previous interactions and successful strategies can be adapted by other players. Also, animals are not limited to only choosing one strategy, but can choose $\mathrm{C}$ 
or D with varying probabilities. Through these changes, Smith and Price were searching for a strategy that once adopted by a majority of players could not be be overtaken by variants of that strategy. This is known as an Evolutionary Stable Strategy or ESS. For understanding how strategies arise and persist in a population, the ESS is more relevant than a Nash equilibrium.

\subsection{Replicator Equations}

In order to find and investigate strategies that are evolutionary stable there must be a dynamical component to the model. The replicator equations are a natural way to model population dynamics with an underlying payoff matrix.

Replicator dynamics assumes that a population is divided into $n$ groups, each utilizing different strategies ranging from $S_{i}$ to $S_{n}$ [6].The fraction or frequency that a group appears in the population is denoted by $x_{1}, \ldots, x_{n}$. Notice that since the $x_{i}$ are frequencies then $\sum x_{i}=1$. The fitness, $f_{i}$, of a strategy $S_{i}$ influences the rate of growth of that population and is determined by the current state of the population $\mathbf{x}=\left\{x_{1}, \ldots, x_{n}\right\}$ and the payoff matrix. That is, if $A$ is the associated payoff matrix, and the entry $a_{i j}$ corresponds to the payoff received by a player playing strategy $S_{i}$ against strategy $S_{j}$ then,

$$
f_{i}(\mathbf{x})=\sum_{j} a_{i j} x_{j}=(A \mathbf{x})_{i}
$$

We further assume that our population is very large, interactions between

all members of the population happen instantaneously, and the evolution of the state $\mathbf{x}(t)$ is continuous. Based on natural selection, we would expect that 
strategies who are more fit will have more reproductive success and become a larger fraction of the population. Therefore, the population dynamics can be modeled by a system of ordinary differential equations (ODEs), known as the replicator equations, which have the following form:

$$
\dot{x}_{i}=x_{i}\left(f_{i}(\mathbf{x})-\bar{f}(\mathbf{x})\right) \quad i=1, \ldots, n
$$

Here, $\bar{f}(\mathbf{x})$ is the average fitness of the population frequency.

$$
\bar{f}(\mathbf{x})=\sum x_{i} f_{i}(\mathbf{x})
$$

In eq. (2.5), when a strategy has a fitness above average, the frequency of that strategy in the population grows. On the other hand, if the fitness is below average then the frequency of that strategy in the population declines. In this way, elements of game theory are combined with differential equations to study the evolution of strategies in a population.

Since we have stipulated that $\sum x_{i}=1$, then the dynamics of the replicator equations lie on the set of non-negative points whose sum is one. This set is known as the simplex [13]. Essentially, the condition that the population frequencies must sum to one reduces the dimension of the system of replicator equations by one. For example, if there were two strategies, the simplex would be a line and if there were three strategies the simplex would be a plane.

The equilibria associated with the replicator equations are connected to the game theory concepts of Nash equilibriums and evolutionary stable strategies. Equilibrium points that are asymptotically stable correspond to an evolutionary stable state, meaning that the population frequencies will not rest at any other nearby state. Also, equilibrium points in the replicator equations which are Lyapunov stable correspond to Nash equilibria [6]. Hence, by analyzing the 
dynamics of the replicator equations for a specific game, we are still able to draw conclusions of a game theoretic nature. 


\section{CHAPTER 3}

\section{ROCK-PAPER-SCISSOR MODEL}

In this work, we wish to consider a game where there are three strategies competing against each other. Each strategy has an advantage over one other strategy, but a disadvantage over the third. This game is most commonly known as the Rock-Paper-Scissor (RPS) game. There are many games that fall under the RPS category, but all of them have a payoff matrix that can be reduced to the following:

$$
\begin{aligned}
& R \quad P \quad S \\
& \begin{array}{l}
R \\
P \\
S
\end{array}\left(\begin{array}{ccc}
0 & -a_{2} & b_{3} \\
b_{1} & 0 & -a_{3} \\
-a_{1} & b_{2} & 0
\end{array}\right)
\end{aligned}
$$

For now, we assume that $a_{i}$ and $b_{i}$ are positive constants. The replicator equations for the matrix (3.1) have been well studied [13] and are known to have three equilibrium points at the vertices of the simplex and one equilibrium in the interior of the simplex. The stability of the interior equilibrium is dependent on determinant of the payoff matrix. If the determinant of (3.1) is positive, which corresponds to $a_{1} a_{2} a_{3}<b_{1} b_{2} b_{3}$, then the interior equilibrium is globally stable. If instead the determinant is negative and $a_{1} a_{2} a_{3}>b_{1} b_{2} b_{3}$, then the interior equilibrium is unstable. In the case where $a_{1} a_{2} a_{3}=b_{1} b_{2} b_{3}$ the determinant is zero and the interior equilibrium point is a center surrounded by neutral oscillations [13].

We are particularly interested in the dynamics for the case where the determinant of (3.1) is zero. For parameters that satisfy this condition, the replicator equations are structurally unstable, meaning that small perturbations to the 
equations can lead to qualitatively different dynamics. Therefore, variations of this RPS model are more interesting to study.

\subsection{Dynamics of Standard RPS Model}

To understand the dynamics of more complicated RPS models such as the timevarying RPS model that is the focus of this work, we must first investigate the dynamics of the simplest RPS model. This model, referred to as the standard RPS model, has all $a_{i}$ equal to 1 [22]. Hence the payoff matrix is the following,

$$
\begin{gathered}
R \\
R \\
P\left(\begin{array}{ccc}
0 & P & S \\
S & -1 & 1 \\
1 & 0 & -1 \\
-1 & 1 & 0
\end{array}\right)
\end{gathered}
$$

Notice that the standard model has a payoff matrix whose determinant is zero. Therefore we should expect that the interior equilibrium point will be a center surrounded by neutral oscillations [13]. Using this payoff matrix (3.2) we can derive the replicator equations which determine the population dynamics of the system. From section 2.3, we know that the replicator equations are defined as,

$$
\dot{x}_{i}=x_{i}\left(f_{i}(\mathbf{x})-\bar{f}(\mathbf{x})\right) \quad i=1, \ldots, n
$$

where,

$$
f_{i}(\mathbf{x})=\sum_{j} a_{i j} x_{j}=(A \mathbf{x})_{i}
$$

and

$$
\bar{f}(\mathbf{x})=\sum x_{i} f_{i}(\mathbf{x})
$$


This gives the following fitnesses,

$$
\begin{aligned}
& f_{1}=-x_{2}+x_{3} \\
& f_{2}=x_{1}-x_{3} \\
& f_{3}=-x_{1}+x_{2}
\end{aligned}
$$

which in turn gives an average fitness of zero.

$$
\bar{f}(\mathbf{x})=x_{1}\left(-x_{2}+x_{3}\right)+x_{2}\left(x_{1}-x_{3}\right)+x_{3}\left(-x_{1}+x_{2}\right)=0
$$

From eq. 3.3 we see that the replicator equations become

$$
\begin{aligned}
& \dot{x_{1}}=x_{1}\left(-x_{2}+x_{3}\right) \\
& \dot{x_{2}}=x_{2}\left(x_{1}-x_{3}\right) \\
& \dot{x_{3}}=x_{3}\left(-x_{1}+x_{2}\right)
\end{aligned}
$$

and we have a three-dimensional, first-order, nonlinear dynamical system. Since $x_{1}, x_{2}, x_{3}$ denote population frequencies, then their sum must equal 1 . This allows us to reduce the dimension of our system down to two by substituting $x_{3}=1-x_{1}-x_{2}$.

$$
\begin{aligned}
& \dot{x_{1}}=x_{1}\left(1-2 x_{2}-x_{1}\right) \\
& \dot{x_{2}}=x_{2}\left(x_{2}+2 x_{1}-1\right)
\end{aligned}
$$

Hence the dynamics can be represented on a two dimensional plane. To find the equilibrium points we set eqs. 3.13-3.14 to zero and solve the system of equations.

$$
\begin{aligned}
& 0=x_{1}\left(1-2 x_{2}-x_{1}\right) \\
& 0=x_{2}\left(x_{2}+2 x_{1}-1\right)
\end{aligned}
$$

Doing so gives four equilibrium points. Three points lie at the corners of the simplex at $(0,0,1),(0,1,0)$, and $(1,0,0)$. The fourth equilibrium is located right 
at the center of the simplex at $\left(\frac{1}{3}, \frac{1}{3}, \frac{1}{3}\right)$. A linear stability analysis shows that the equilibrium points at the corners of the simplex are saddle points, while the interior equilibrium point is a linear center. Furthermore, eq. 3.13 and eq. 3.14 admit a first integral.

$$
x_{1} x_{2}\left(1-x_{1}-x_{2}\right)=\text { constant }
$$

When the integral curves are plotted in figure 3.1, we see that there is indeed a center at $\left(\frac{1}{3}, \frac{1}{3}, \frac{1}{3}\right)$ surrounded by neutral cycles. Therefore, in the interior of simplex and away from the center point, the population frequencies change in time and no one strategy reaches a stable state.

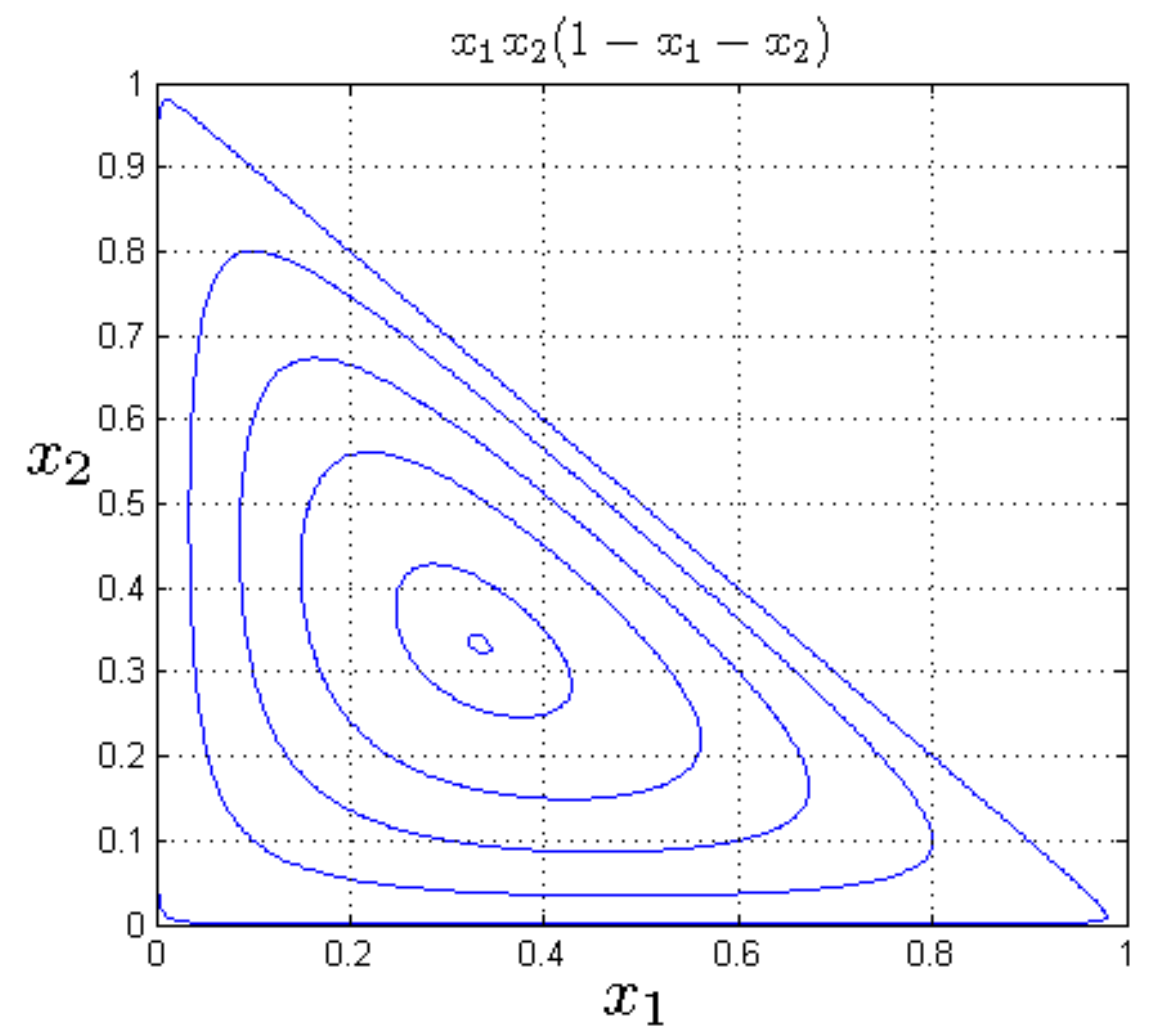

Figure 3.1: Solutions to the standard RPS model plotted in two dimensions. The values of $x_{3}$ can be found by using $x_{3}=1-x_{1}-x_{2}$. As expected, there is an equilibrium at $x_{1}=x_{2}=x_{3}=\frac{1}{3}$ and it is a center surrounded by neutral oscillations. 
The standard model is a very special case in the family of RPS models. Any slight perturbation leads to qualitatively different results. Therefore it is an interesting case to study and a good launching point to investigate more complicated RPS models. The model that is the main focus of this work and introduced in chapter 4 simplifies to the standard model for certain choices of parameters and can in fact be considered a perturbation on the standard model for small values of those parameters.

\subsection{Applications of RPS models}

RPS models have been used in the biological and social sciences as a means to understand biodiversity and co-evolution. They apply to systems where there are three competing strategies or populations and the relationship between the strategies is similar to the children's game Rock-Paper-Scissors. Systems that exhibit this relationship will have population frequencies that periodically oscillate.

One example of a biological system that exhibits a rock-paper-scissor dynamic is the male population of the side-blotched lizard or Uta stansburiana [18]. Males of the side-blotched lizard have three different colorings on their throats that correspond to the reproductive strategy they utilize. The males with orange throats have a higher level of testosterone and defend large territories with a large number of females. Blue-colored males have lower testosterone and defend smaller territories with less females. The third type is referred to as a "sneaker" male. It has a yellow-colored throat which resembles the female side-blotch lizard. Since it appears similar to female side-blotch lizards it is able 
to sneak into large territories undetected and mate with the females in that territory. Hence the "sneaker" male had an advantage over the orange-colored male with the large territory, but not over the blue-colored male with the small territory since the blue-colored male can more easily defend his territory. However, the orange-colored male has an advantage over the blue-colored male since he has access to a larger number of females due to maintaining a larger territory. Thus, the three reproductive strategies compete in a rock-paper-scissor fashion. In a paper titled "The rock-paper-scissor game and the evolution of alternative male strategies," Sinervo and Lively were able to show that the populations of the different male types did in fact oscillate with one strategy dominating for a time before it was overtaken by the strategy that has an advantage over it [18].

Another example of a biological system with a rock-paper-scissor dynamic is the interaction between three competing strains of Escherichia coli, otherwise known as E. coli. According to Kirkup and Riley in "Antibiotic-mediated antagonism leads to a bacterial game of rock-paper-scissor in vivo," there exist a strain of E. coli that produces colicins, which are a type of antibiotic. The antibioticproducing strain kills another strain of E. coli that is sensitive to the antibiotic. However a third strain exists that is resistant to the antibiotic and out competes the antibiotic strain. When no antibiotic strain is present, the sensitive strain out competes the resistant strain. Thus these three strains exhibit a rock-paperscissor dynamic. In their paper, Kirkup and Riley where able to show that all three strains of E. coli could coexist as long as their environment were spatially structured [8].

Rock-paper-scissor dynamics also occur in fields outside of biology. In a paper by Semmann, Krambeck, and Milinski, the authors give an example 
of a sociological system with three strategies whose populations oscillate in a RPS manner [17]. They conducted public goods experiments where people could anonymously choose from three strategies for an undetermined number of rounds. Subjects could choose the cooperation strategy which meant that they joined a group and contributed money to a public pool that is then multiplied by a factor larger than 1 and distributed evenly amongst members of the group. Subjects could also choose to join the group and then defect by not contributing any money. In this way, they receive the benefit of the distributed money without any of the cost. The third strategy referred to as the "loner" strategy does not join the group and instead receives a fixed amount money. If the population is composed of mostly cooperators then the defectors have an advantage since they receive the group benefit without the cost. However, if the population consists primarily of defectors then money distributed to the group will be very low and loners have the advantage. Finally, in a population composed of mostly loners, cooperators can potentially receive more money and gain an advantage. Similar to the previous papers, the authors found that the populations of cooperators, defectors, and loners oscillated so that whichever strategy was most abundant would be overtaken by the strategy which has an advantage over it.

Due to the number of real-life applications, there are number of papers that study variations of the RPS model hoping to capture a certain type of behavior or understand mechanisms for biodiversity. For example, Mauro Mobilia [11] introduced mutation into the RPS model and found that for low mutation rates there exists parameters for which the system produces a limit cycle as a result of a Hopf bifurcation. In this work, we seek to investigate a model that adds a periodic forcing term to the standard RPS model. One application could be 
to model the effect of seasonal variation and its impact on the payoffs of the competing strategies. 


\section{CHAPTER 4}

\section{RPS MODEL WITH PERIODIC TIME-VARYING PAYOFFS - A SIMPLE \\ CASE}

\subsection{Model}

We are interested in considering a variation of the RPS model that has entries in the payoff matrix that are time-dependent and periodic. Our motivation is to create a model that will account for possible seasonal variation in the interactions of the strategies. Previous work done by Rand et al. [15] considers a variation of the RPS model with two periodic terms added to the standard model. Our model expands on that work and considers the addition of periodic terms to all nonzero entries of the payoff matrix. We therefore propose a model of the following form:

$$
R\left(\begin{array}{ccc}
R & P & S \\
P & -1+A_{1} \cos \omega t & 1+A_{2} \cos \omega t \\
0 & 0 & -1+A_{4} \cos \omega t \\
1+A_{3} \cos \omega t & 0
\end{array}\right)
$$

Once we eliminate $x_{3}$ by applying the constraint $x_{3}=1-x_{1}-x_{2}$, the new RSP model leads to replicator equations of the following type,

$$
\begin{aligned}
& \dot{x_{1}}=x_{1}\left(1-2 x_{2}-x_{1}\right)+x_{1} G_{1}\left(x_{1}, x_{2} ; A_{i}\right) \cos \omega t \\
& \dot{x_{2}}=x_{2}\left(x_{2}+2 x_{1}-1\right)+x_{2} G_{2}\left(x_{1}, x_{2} ; A_{i}\right) \cos \omega t
\end{aligned}
$$

where $G_{1}$ and $G_{2}$ are polynomials in $x_{1}, x_{2}$, and the $A_{i}{ }^{\prime}$ s.

The presence of the time-varying periodic terms, $A_{i} \cos \omega t$, destroys the first integral found in the original RPS model. In addition, for general values of $A_{i}$, the equilibrium at $\left(\frac{1}{3}, \frac{1}{3}\right)$ disappears. 


\subsection{Simple Case}

We begin our analysis of the proposed model by first studying the model put forth by Rand et al. [15] If we set $A_{1}=-A_{2}=-A$ and $A_{3}=A_{4}=A_{5}=A_{6}=0$ then our payoff matrix becomes:

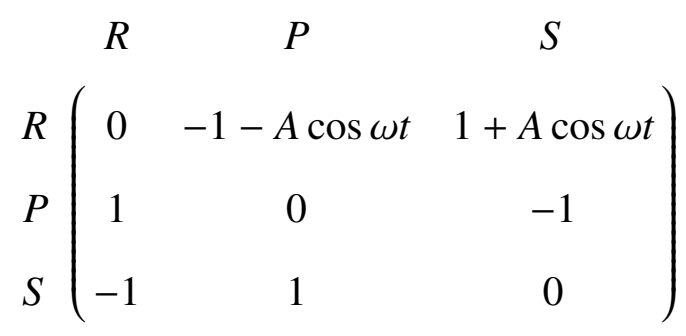

and the corresponding replicator equations are:

$$
\begin{aligned}
& \dot{x_{1}}=x_{1}\left(1-2 x_{2}-x_{1}\right)\left[1+\left(1-x_{1}\right) A \cos \omega t\right] \\
& \dot{x_{2}}=x_{2}\left(x_{2}+2 x_{1}-1+\left[x_{1}\left(2 x_{2}+x_{1}-1\right)\right] A \cos \omega t\right)
\end{aligned}
$$

This is exactly the model introduced by Rand et al. [15] Numerical integration shows that for small values of $A$ the periodic motions of the standard RPS system are typically replaced by quasiperiodic motions, see figure 4.1. In particular, motions starting near the equilibrium point $\left(\frac{1}{3}, \frac{1}{3}\right)$ typically remain near it as seen in figure 4.1. An exception occurs for certain values of the system parameters $A$ and $\omega$. See figure 4.2 which displays a numerically integrated motion starting near $\left(\frac{1}{3}, \frac{1}{3}\right)$ for parameters $A=0.1, \omega=1.154$. Note that here a motion which starts near the equilibrium $\left(\frac{1}{3}, \frac{1}{3}\right)$ travels far away from it.

In a previous work done by Rand et al. [15], resonant values of the parameters $\omega$ and $A$ were identified using Floquet theory and associated instabilities of the equations linearized about the equilibrium point were studied. Here we seek to explain the phenomenon in figure 4.1 and figure 4.2 through the use of perturbation methods. This will allow us to understand non only where such 


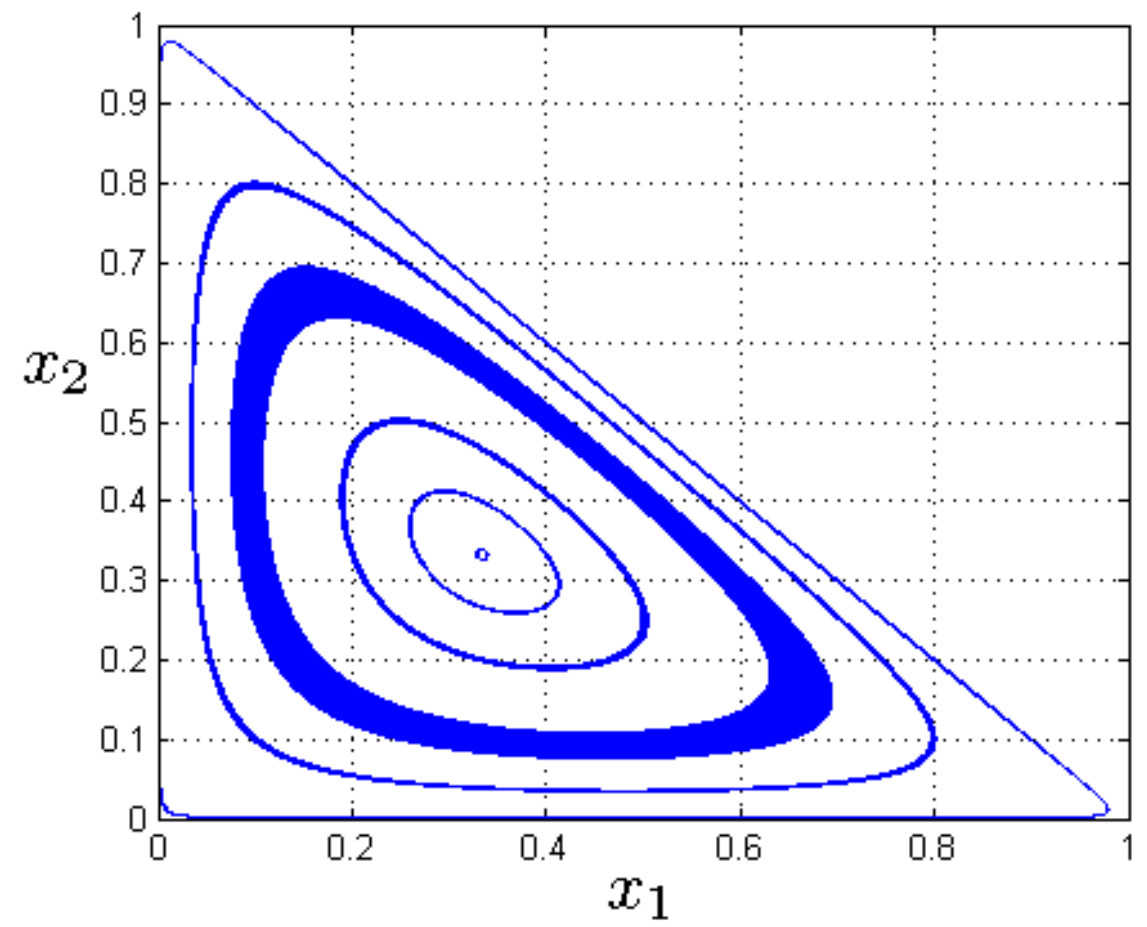

Figure 4.1: Motions of eqs. (4.5), (4.6) for $A=0.02$ and $\omega=1$ obtained by numerical integration. Here the periodic motions of figure 3.1 are replaced by quasiperiodic motions. Note that motions starting near the equilibrium point $\left(\frac{1}{3}, \frac{1}{3}\right)$ remain near it.

resonances occur, but also the structure of the phase space in the neighborhood of parametric resonances.

\subsection{Linear Resonance}

We begin by translating the origin to the equilibrium at $\left(\frac{1}{3}, \frac{1}{3}\right)$ and scaling the coordinates by $\epsilon<<1$. We set

$$
x_{1}=\epsilon x+\frac{1}{3}, \quad x_{2}=\epsilon y+\frac{1}{3},
$$




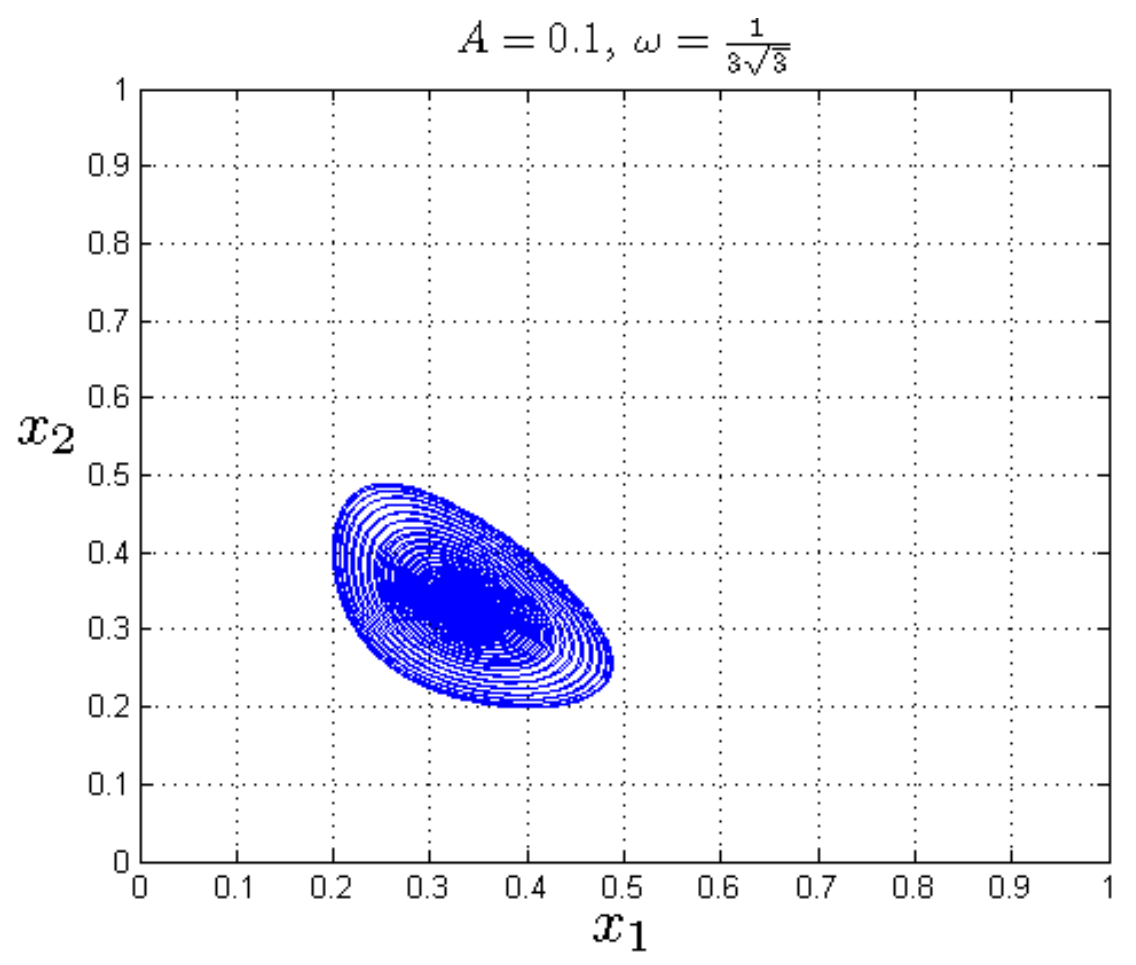

Figure 4.2: Motion of eqs. (4.5), (4.6) for $A=0.1$ and $\omega=1.154$ for initial conditions $x_{1}=x_{2}=0.333$ obtained by numerical integration. Note that here a motion which starts near the equilibrium $\left(\frac{1}{3}, \frac{1}{3}\right)$ travels far away from it.

and substitute these into eqs.(4.5) and(4.6), giving:

$$
\begin{aligned}
& \dot{x}=\frac{(3 \epsilon x+1)((3 \epsilon x-2) A \cos \omega t-3)(2 y+x)}{9} \\
& \dot{y}=\frac{(3 \epsilon y+1)\left(\left(6 \epsilon x y+2 y+3 \epsilon x^{2}+x\right) A \cos \omega t+6 x+3 y\right)}{9}
\end{aligned}
$$

Our first step in the analysis of these ODEs is to determine which values of $\omega$ produce instability via parametric resonance for small values of the forcing amplitude $A$. In the work by Rand et al.[15], this was accomplished by using Floquet theory. Here we obtain this information directly from the perturbation method as follows. We first linearize (4.8),(4.9) for small values of $x$ and $y$. This 
can be done by setting $\epsilon=0$, giving:

$$
\begin{aligned}
& \dot{x}=-\left(\frac{x+2 y}{3}\right)-\frac{2}{9}(2 y+x) A \cos \omega t \\
& \dot{y}=\left(\frac{2 x+y}{3}\right)+\frac{1}{9}(2 y+x) A \cos \omega t
\end{aligned}
$$

Now we look for a solution to these equations via regular perturbations, valid for small $A<<1$. The simplest way to do this is to transform this first order system of ODEs into a single second order ODE by differentiating (4.10) and substituting expressions for $\dot{y}$ from (4.11) and for $y$ from (4.10), giving:

$$
f_{1} \ddot{x}+f_{2} \dot{x}+f_{3} x=0
$$

where

$$
\begin{aligned}
& f_{1}=3+2 A \cos \omega t \\
& f_{2}=2 A \omega \sin \omega t \\
& f_{3}=\left(\frac{3+2 A \cos \omega t}{3}\right)^{2}
\end{aligned}
$$

We set

$$
x=x_{0}+A x_{1}+O\left(A^{2}\right)
$$

Substituting (4.16) into (4.12) and collecting terms gives:

$$
\begin{aligned}
& \ddot{x}_{0}+\frac{x_{0}}{3}=0 \\
& \ddot{x}_{1}+\frac{x_{1}}{3}=-\frac{2}{3} \ddot{x}_{0} \cos \omega t-\frac{2}{3} \omega \dot{x}_{0} \sin \omega t-\frac{4}{9} x_{0} \cos \omega t
\end{aligned}
$$

Eq.(4.17) shows that $x_{0}$ will have frequency $1 / \sqrt{3}$, whereupon the right hand side of eq.(4.18) will have terms with frequencies:

$$
\omega \pm \frac{1}{\sqrt{3}}
$$

Resonant values of $\omega$ will correspond to forcing frequencies (4.19) which are equal to natural frequencies of the homogeneous $x_{1}$ equation, i.e. to $1 / \sqrt{3}$. This 
gives that

$$
\omega=\frac{2}{\sqrt{3}} \quad \text { (resonance) }
$$

This value of $\omega$ corresponds to the largest resonance tongue. There are an infinitude of smaller tongues which would emerge from the perturbation method if we were to continue it to $O\left(A^{2}\right)$ and higher. These have been shown to be of the form $\omega=2 /(n \sqrt{3})$ for $n=2,3, \ldots[15]$.

\subsection{Multiple Scales Perturbation Method}

The resonance (4.20) partially explains the phenomenon displayed in figure 4.2: when $\omega$ lies close to the resonant value of $\frac{2}{\sqrt{3}}$, motions which start near the equilibrium point $(x=0, y=0)$ (i.e. $\left.\left(x_{1}=\frac{1}{3}, x_{2}=\frac{1}{3}\right)\right)$ may move relatively far away from it. This result is incomplete in that it does not explain how far a motion will travel from the equilibrium point, how the motion depends on initial conditions, or how close to the resonance value (4.20) the parameter $\omega$ must be chosen for this phenomenon to occur. Here we obtain approximate answers to these questions by using a more powerful perturbation approach.

We prepare for the perturbation expansion by setting $\tau=\omega t$ and $A=\epsilon^{2}$ in eqs. (4.8), (4.9), and then again transforming the two first order ODEs into a single second order nonlinear ODE, giving:

$$
\begin{aligned}
& 18 \omega^{2}(3 \epsilon x+1)\left(3 \epsilon^{3} x \cos \tau-2 \epsilon^{2} \cos \tau-3\right) x^{\prime \prime}= \\
& 81 \epsilon \omega^{2}\left(6 \epsilon^{3} x \cos \tau-1\right) x^{\prime 2}-18 \epsilon^{2} \omega^{2} \sin \tau(3 \epsilon x-2)(3 \epsilon x+1) x^{\prime} \\
& \quad-243 \epsilon^{9} x^{6} \cos ^{2} \tau+162 \epsilon^{6} \cos \tau\left(2 \epsilon^{2} \cos \tau+3\right) x^{5}
\end{aligned}
$$




$$
\begin{aligned}
& -27 \epsilon^{3}\left(\epsilon^{4} \cos ^{2} \tau+12 \epsilon^{2} \cos \tau+9\right) x^{4}-18 \epsilon^{4} \cos \tau\left(5 \epsilon^{2} \cos \tau+9\right) x^{3} \\
& +3 \epsilon\left(2 \epsilon^{2} \cos \tau+3\right)\left(2 \epsilon^{2} \cos \tau+9\right) x^{2}+2\left(2 \epsilon^{2} \cos \tau+3\right)^{2} x
\end{aligned}
$$

Here primes represent differentiation with respect to $\tau$. Neglecting terms of $O\left(\epsilon^{3}\right)$, we obtain

$$
\begin{aligned}
\omega^{2} x^{\prime \prime}+\frac{x}{3}= & \frac{\epsilon\left(3 \omega^{2} x^{\prime 2}-x^{2}\right)}{2} \\
& -\frac{\epsilon^{2}\left(\omega^{2}\left(81 x x^{\prime 2}+12 x^{\prime} \sin \tau\right)-27 x^{3}+4 x \cos \tau\right)}{18}+O\left(\epsilon^{3}\right)(
\end{aligned}
$$

Next we define three time scales $\xi, \eta$ and $\zeta$ :

$$
\xi=\tau, \quad \eta=\epsilon \tau, \quad \zeta=\epsilon^{2} \tau
$$

and we consider $x$ to be a function of $\xi, \eta$ and $\zeta$, whereupon the chain rule gives:

$$
\begin{aligned}
x^{\prime} & =x_{\xi}+\epsilon x_{\eta}+\epsilon^{2} x_{\zeta} \\
x^{\prime \prime} & =x_{\xi \xi}+2 \epsilon x_{\xi \eta}+2 \epsilon^{2} x_{\xi \zeta}+\epsilon^{2} x_{\eta \eta}
\end{aligned}
$$

We detune $\omega$ off of the resonance (4.20):

$$
\omega=\frac{2}{\sqrt{3}}+k \epsilon^{2}+\cdots
$$

and expand $x=x_{0}+\epsilon x_{1}+\epsilon^{2} x_{2}+\cdots$. Substituting (4.24),(4.25) and these expansions into (4.22) and collecting terms, we obtain:

$$
\begin{gathered}
L x_{0}=0, \quad \text { where } L(\cdot)=(\cdot)_{\xi \xi}+\frac{1}{4}(\cdot) \\
L x_{1}=-\frac{3}{8} x_{0}^{2}-2 x_{0 \xi \eta}+\frac{3}{2} x_{0 \xi}^{2} \\
L x_{2}=-\frac{3}{4} x_{0} x_{1}+\frac{9}{8} x_{0}^{3}-\sqrt{3} k x_{0 \xi \xi}-\frac{1}{6} x_{0} \cos \xi-2 x_{1 \xi \eta} \\
-2 x_{0 \xi \zeta}-x_{0 \eta \eta}+3 x_{0 \xi} x_{1 \xi}-\frac{9}{2} x_{0} x_{0 \xi}^{2}-\frac{2}{3} x_{0 \xi} \sin \xi+3 x_{0 \eta} x_{0 \xi}
\end{gathered}
$$


We take the solution of (4.27) in the form:

$$
x_{0}=a_{0}(\eta, \zeta) \cos \frac{\xi}{2}+b_{0}(\eta, \zeta) \sin \frac{\xi}{2}
$$

Substituting the expression for $x_{0}$ (4.30) into the $x_{1}$ equation (4.28), and removing secular terms gives

$$
\frac{\partial a_{0}}{\partial \eta}=0, \quad \frac{\partial b_{0}}{\partial \eta}=0 \Rightarrow a_{0}=a_{0}(\zeta), b_{0}=b_{0}(\zeta)
$$

Solving for $x_{1}$, we obtain

$$
x_{1}=a_{1}(\eta, \zeta) \cos \frac{\xi}{2}+b_{1}(\eta, \zeta) \sin \frac{\xi}{2}+a_{0} b_{0} \sin \xi-\frac{1}{2} b_{0}^{2} \cos \xi+\frac{1}{2} a_{0}^{2} \cos \xi
$$

Next we substitute the expression for $x_{1}$ (4.32) into the $x_{2}$ equation (4.29), and remove secular terms, giving

$$
\frac{\partial a_{1}}{\partial \eta}=f\left(a_{0}, b_{0}\right), \quad \frac{\partial b_{1}}{\partial \eta}=g\left(a_{0}, b_{0}\right)
$$

where

$$
\begin{aligned}
& f\left(a_{0}, b_{0}\right)=-\frac{d a_{0}}{d \zeta}+\frac{b_{0}}{12}-\frac{\sqrt{3}}{4} k b_{0}-\frac{3}{4} b_{0}^{3}-\frac{3}{4} a_{0}^{2} b_{0} \\
& g\left(a_{0}, b_{0}\right)=-\frac{d b_{0}}{d \zeta}+\frac{a_{0}}{12}+\frac{\sqrt{3}}{4} k a_{0}+\frac{3}{4} a_{0}^{3}+\frac{3}{4} b_{0}^{2} a_{0}
\end{aligned}
$$

Now from eq.(4.31) we see that $a_{0}$ and $b_{0}$ do not depend on $\eta$ and thus neither do $f\left(a_{0}, b_{0}\right)$ or $g\left(a_{0}, b_{0}\right)$. Eqs.(4.33) show that $a_{1}$ and $b_{1}$ will grow linearly in time $\eta$ unless $f\left(a_{0}, b_{0}\right)=0$ and $g\left(a_{0}, b_{0}\right)=0$. Thus for $a_{1}$ and $b_{1}$ to remain bounded, we require

$$
\begin{aligned}
& \frac{d a_{0}}{d \zeta}=\frac{b_{0}}{12}-\frac{\sqrt{3}}{4} k b_{0}-\frac{3}{4} b_{0}^{3}-\frac{3}{4} a_{0}^{2} b_{0} \\
& \frac{d b_{0}}{d \zeta}=\frac{a_{0}}{12}+\frac{\sqrt{3}}{4} k a_{0}+\frac{3}{4} a_{0}^{3}+\frac{3}{4} b_{0}^{2} a_{0}
\end{aligned}
$$

This system of slow-flow equations is easier to study in polar coordinates so we set $a_{0}=r \cos \theta$ and $b_{0}=r \sin \theta$. The slow flow equations become:

$$
\frac{\partial r}{\partial \zeta}=\frac{1}{12} r \sin 2 \theta, \quad \frac{\partial \theta}{\partial \zeta}=\frac{3}{4} r^{2}+\frac{\cos 2 \theta}{12}+\frac{\sqrt{3}}{4} k
$$


In view of eq.(4.30), equilibrium points in the slow-flow (4.38) correspond to periodic motions in the standard RPS system (4.5), (4.6). The first of (4.38) gives $\theta=0, \pi / 2, \pi, 3 \pi / 2$, whereupon the second of (4.38) gives

$$
\frac{3}{4} r^{2} \pm \frac{1}{12}+\frac{\sqrt{3}}{4} k=0 \Rightarrow r^{2}=\mp \frac{1}{9}-\frac{\sqrt{3}}{3} k
$$

Since $r^{2}>0$, we get bifurcations at

$$
k= \pm \frac{1}{3 \sqrt{3}}
$$

For $k>\frac{1}{3 \sqrt{3}}$ there are no nontrivial equilibria, while for $k<-\frac{1}{3 \sqrt{3}}$ there are four. In the intermediate case of $-\frac{1}{3 \sqrt{3}}<k<\frac{1}{3 \sqrt{3}}$ there are two nontrivial equilibria. Since $A=\epsilon^{2}$ and eq.(4.26), the bifurcation curves have the form:

$$
\omega=\frac{2}{\sqrt{3}} \pm \frac{1}{3 \sqrt{3}} A+\cdots
$$

This agrees with the work done by Rand et al. [15] concerning the location of stability transition curves, see figure 4.3. The slow-flow (4.38) is conservative and admits the following first integral:

$$
9 r^{4}+2(3 \sqrt{3} k+\cos 2 \theta) r^{2}=\text { constant }
$$

Figure 4.4 displays the first integral (4.42) for $k=\frac{1}{3 \sqrt{3}}+0.2=0.3925, k=0$, and $k=-\frac{1}{3 \sqrt{3}}-0.2=-0.3925$, respectively. Here we have identified $x$ with $a_{0}$, being approximately $x_{0}$ at $\xi=0$ in eq.(4.30). Similarly, $x^{\prime}$ is identified with $b_{0} / 2$.

\subsection{Poincaré map}

The foregoing results of the perturbation method may be compared to numerical integration of equation (4.21) by use of a Poincaré map. Here we imagine a 


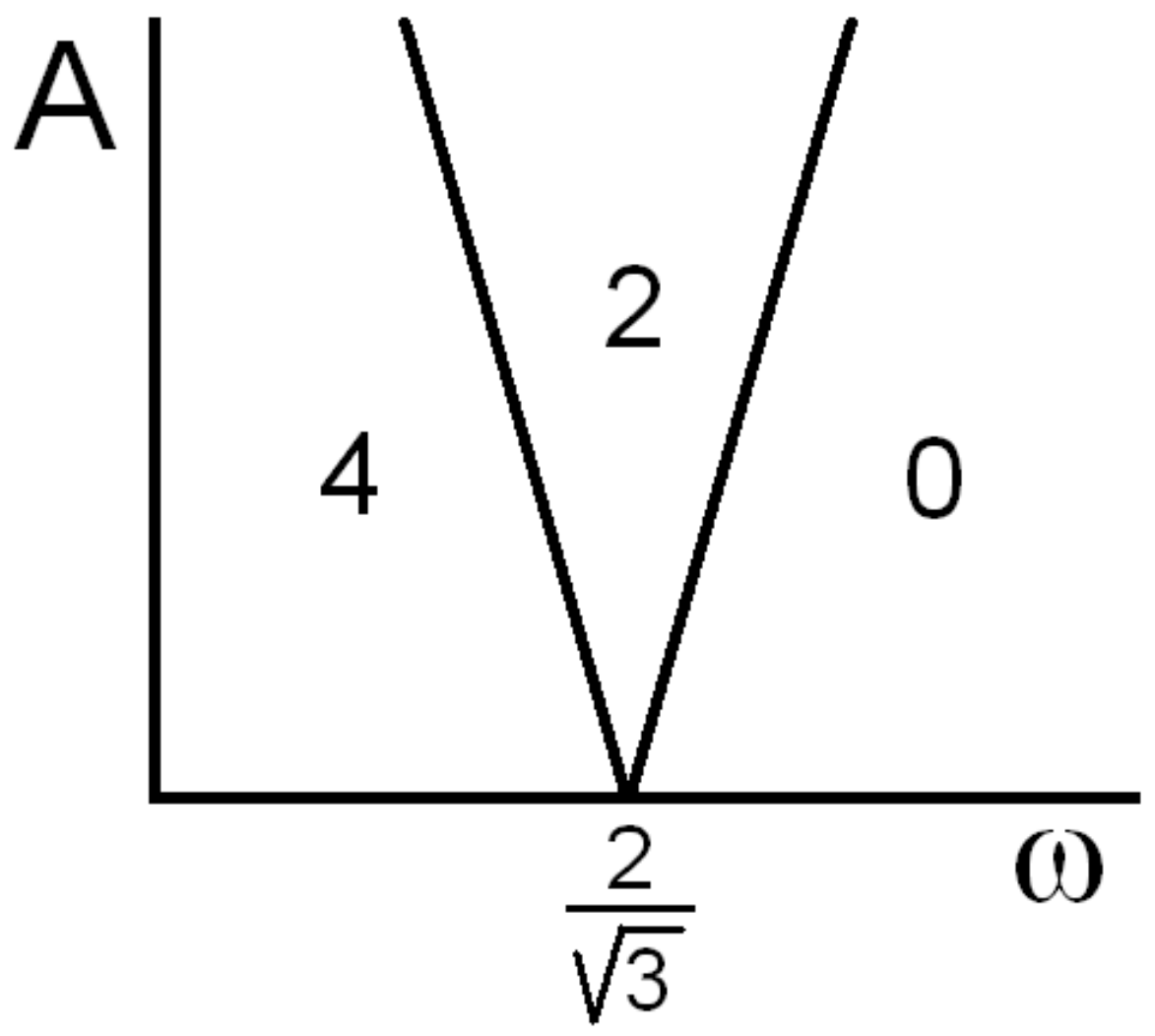

Figure 4.3: Bifurcation curves (4.41) showing the number of nontrivial equilibria in the slow flow (4.36),(4.37). See figure 4.4 for sample phase portraits of the slow flow in each of these regions.

flow on a three-dimensional phase space with axes $x, x^{\prime}, t$, and an associated map produced by sampling the said flow at times $\tau=2 N \pi$, for $N=0,1,2,3, \cdots$. The associated Poincaré maps depend upon both $\omega$ and $\epsilon$. Local behavior around the equilibrium point at the origin $x=x^{\prime}=0$ is naturally affected by $\omega$ as in figure 4.4. The parameter $\epsilon$ affects both the strength of the forcing (because the forcing amplitude $A=\epsilon^{2}$ ) and the importance of nonlinearities (because the coordinates have been scaled by $\epsilon$, cf. eq.(4.7)).

As a check on the perturbation results (which are expected to be valid for small $\epsilon$ ), we first present Poincaré maps for $\epsilon=0.1$ and for the same values 

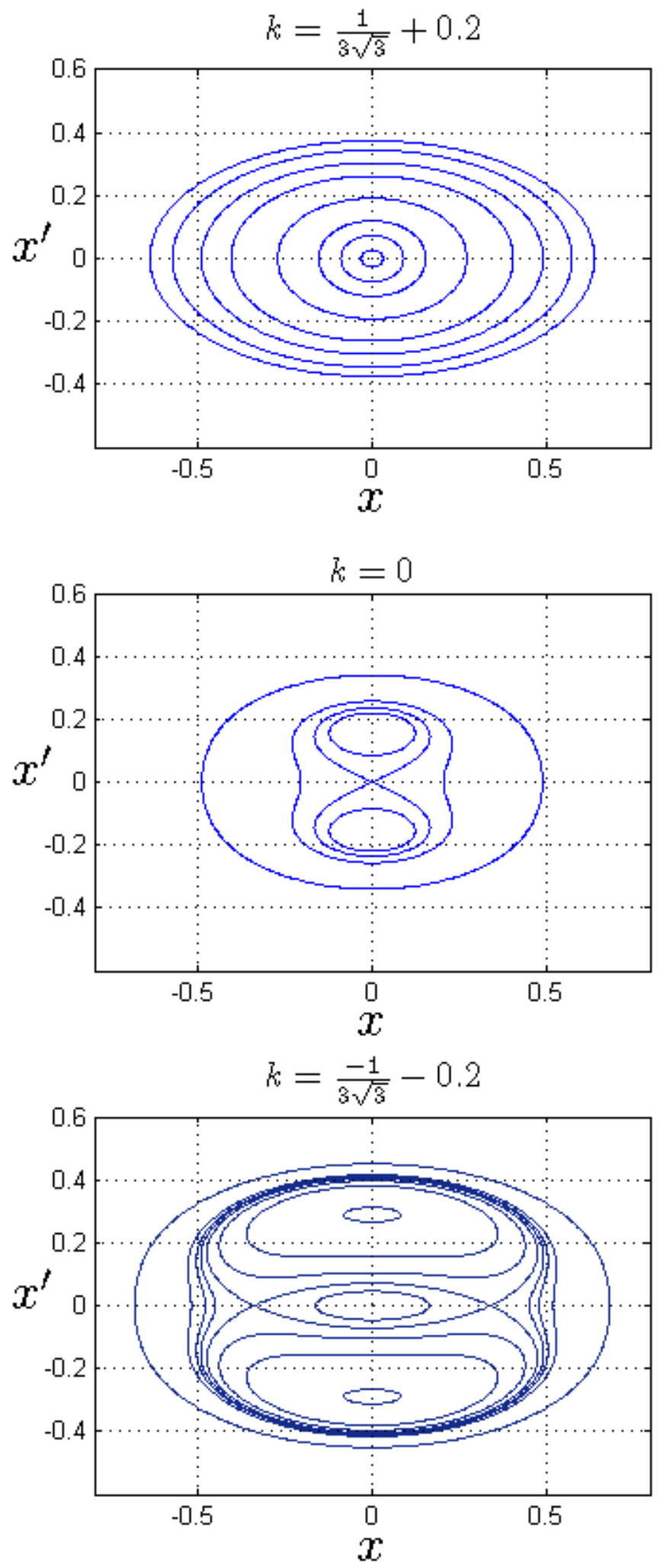

Figure 4.4: Plot of the first integral (4.42) for various values of $k$. Note, that as $k$ decreases, the system traverses the tongue in figure 4.3 from right to left ana9the number of nontrivial equilibria changes. 
of $\omega$ as in figure 4.4. See figure 4.5. Note that there is good agreement in the neighborhood of the origin.

As an example of the kind of behavior which occurs for larger values of $\epsilon$, we present Poincaré maps for $\epsilon=1$ and for the same values of $k$ as in figure 4.5. See figure 4.6. These figures show the appearance of chaos which is associated with KAM theory [1]. KAM theory, named for it's inventors, Kolmogorov, Arnold and Moser, describes the onset of chaos in a perturbed Hamiltonian system. Among the various features of KAM theory is the phenomenon that chaos occurs most noticeably in the neighborhood of motions which in the unperturbed Hamiltonian system are in low order resonance with the periodic driver. This is relevant to us here because eqs. (4.5), (4.6) may be written in the form of a perturbed Hamiltonian system:

$$
\begin{aligned}
& \dot{x}_{1}=\frac{\partial H}{\partial x_{2}}+\frac{A \cos \omega t}{h} \frac{\partial M}{\partial x_{2}} \\
& \dot{x_{2}}=-\frac{\partial H}{\partial x_{1}}-\frac{A \cos \omega t}{h} \frac{\partial M}{\partial x_{1}}
\end{aligned}
$$

where

$$
\begin{aligned}
H & =x_{1} x_{2}\left(1-x_{1}-x_{2}\right) \\
h & =\frac{-1}{x_{1}\left(1-x_{1}\right)^{2}\left(1-x_{1}-2 x_{2}\right)} \\
M & =\frac{x_{2}}{x_{1}-1}
\end{aligned}
$$

Thus when $A=0$, the system (4.5), (4.6) is integrable and the Poincaré map consists of closed curves as shown in figure 3.1. Then as predicted by KAM theory, the closed curves in the Poincaré map of the unperturbed Hamiltonian system which are in $n: 1$ resonance with $\cos \omega t$ are replaced by $2 n$-cycles, one stable and one unstable. The stable $n$-cycle appears in simulations as $n$ closed curves 

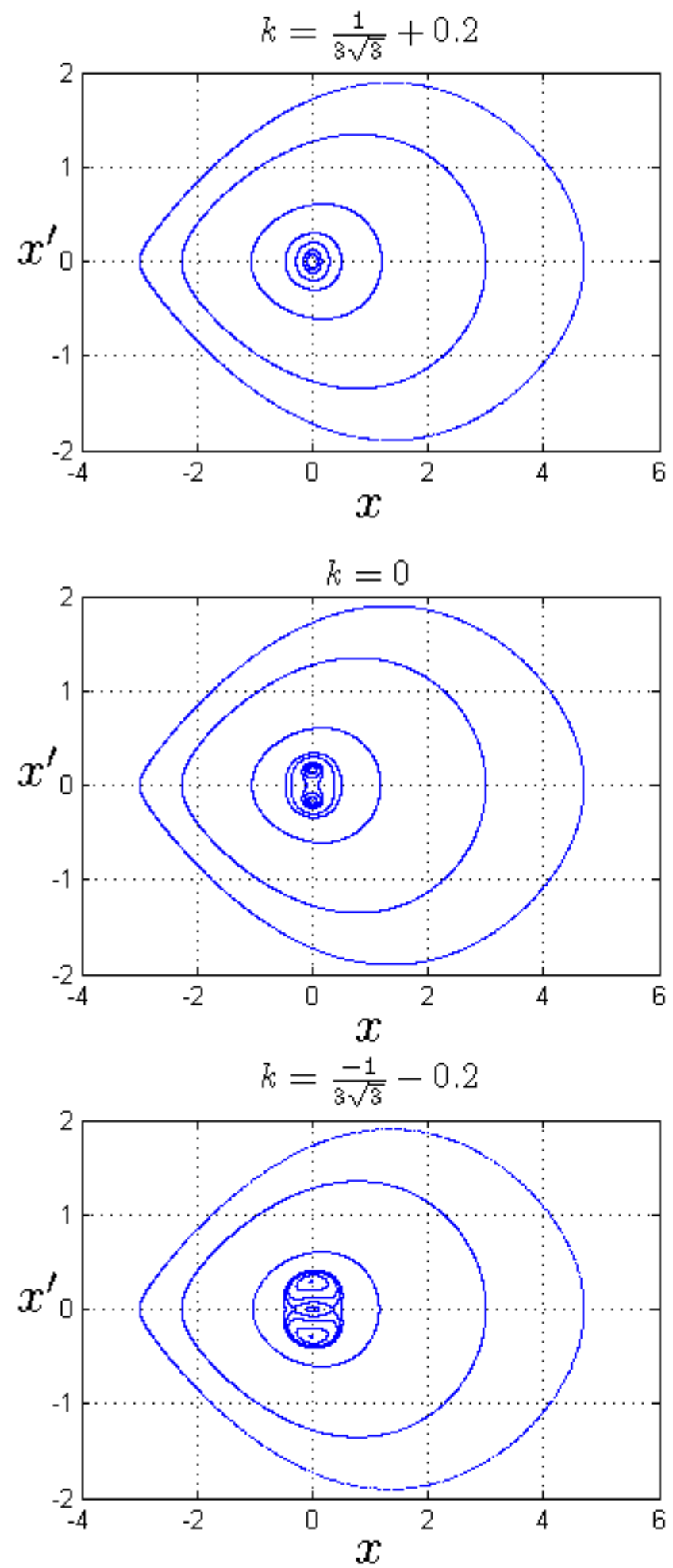

Figure 4.5: Poincaré map obtained by numerically integrating eq.(4.21) for $\epsilon=0.1$ and $\omega=2 / \sqrt{3}+k \epsilon^{2}$. Cf. figure 4.4 . 
lying in the neighborhood of the unperturbed resonant curve. The unstable $n$ cycle appears as $n$ saddles, each carrying a region of localized chaos with it. Some of these features may be seen in figure 4.6.

\subsection{Conclusions}

In this chapter we have investigated a simple case of adding periodic coefficients to a system which is more commonly treated as having constant coefficients. The system studied is a replicator equation based on an RPS scenario characterized by the payoff matrix (4.4) and governed by the differential equations (4.5), (4.6). In the $A=0$ constant coefficient case, this system is integrable with the first integral (3.17) and has the property that the equilibrium at $\left(\frac{1}{3}, \frac{1}{3}\right)$ is Liapunov stable, figure 3.1. By contrast, in the $A>0$ system with periodic forcing, this same equilibrium can be unstable, figure 4.2 , due to parametric resonance, figure 4.3. The analysis presented in this paper, valid for small values of $A$, has shown that detuning off of this resonance is, however, asymmetric. That is, systems which lie outside and just to the left of the resonance tongue of figure 4.3 have very different behavior from those systems which lie just to the right of the same tongue. See figures $4.7,4.8$. This behavior is predicted by the perturbation theory, cf. the Poincaré maps in figure 4.4. For larger values of $A$ we have seen that the system studied exhibits KAM type chaos, figure 4.6.

Our results have implications for biological systems with RPS characteristics, such as the side-blotched lizard Uta stansburiana that displays persistent oscillations in population frequencies [18]. Previous work has described the dynamics

of this species using an evolutionary model with damping, and has attributed 

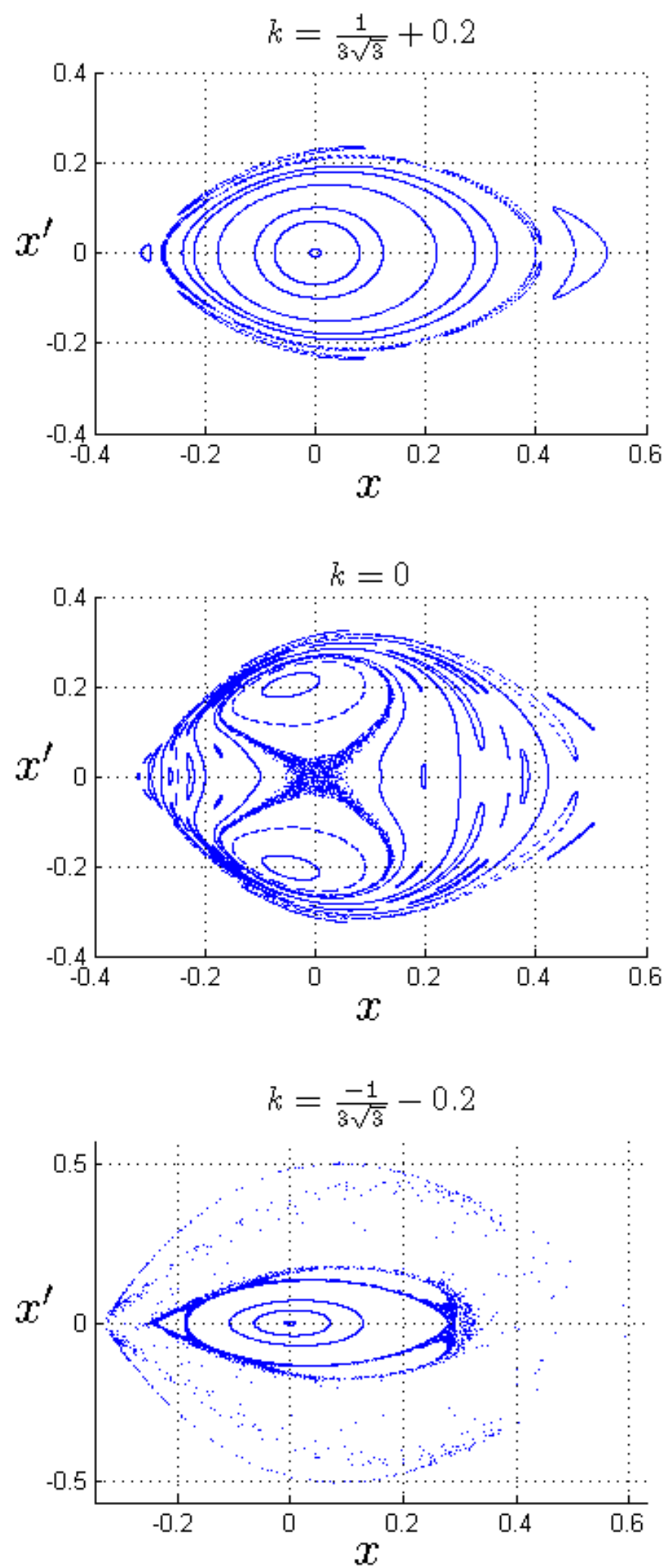

Figure 4.6: Poincaré map obtained by numerically integrating eq.(4.21) for $\epsilon=1$ and $\omega=2 / \sqrt{3}+k \epsilon^{23}$. . ff. figure 4.5 . 
the persistence of the oscillations observed in the data to stochastic perturbations which reset the initial conditions (using a verbal rather than mathematical argument) [18]. In this work, we have instead approached this issue by considering an external forcing function that drives the system via periodicity in the payoff coefficients. We showed how deterministic external forcing can lead to aperiodic variation in population frequency.

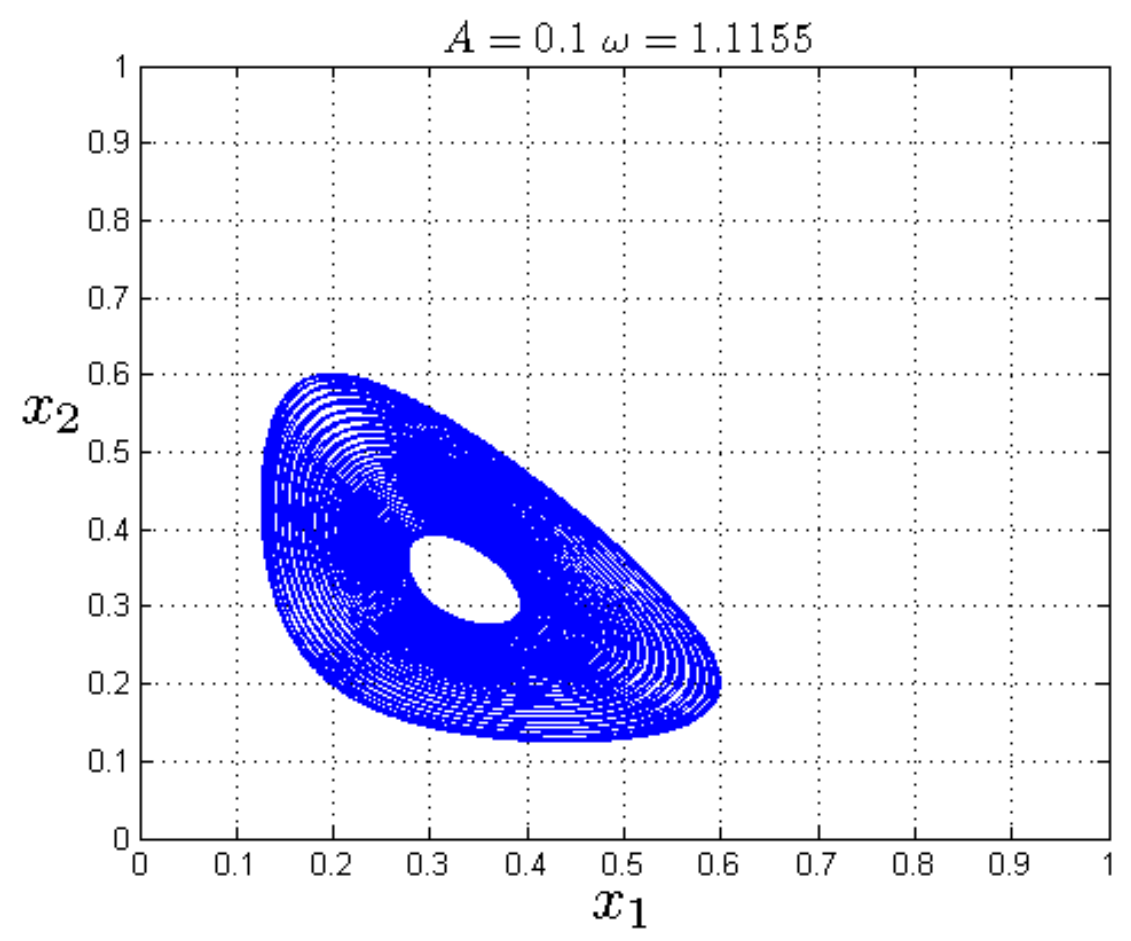

Figure 4.7: Motion of eqs. (4.5), (4.6) for $A=0.1$ and $\omega=\frac{2}{\sqrt{3}}-\left(\frac{1}{3 \sqrt{3}}+0.2\right) A=$ 1.1155 for initial conditions $x_{1}=x_{2}=0.3$ obtained by numerical integration. This system lies to the left of the resonance tongue in figure 4.3. Note that here a motion which starts near the equilibrium $\left(\frac{1}{3}, \frac{1}{3}\right)$ travels far away from it. 


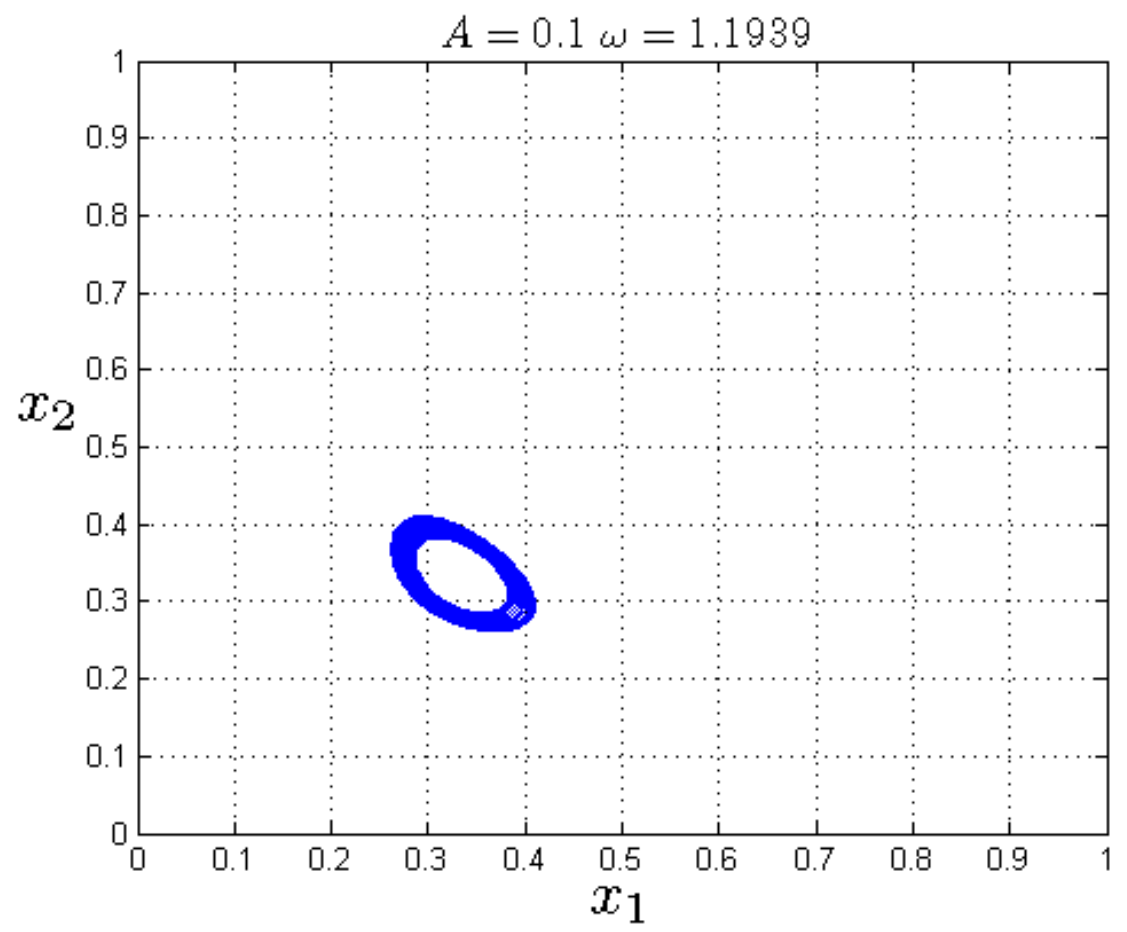

Figure 4.8: Motion of eqs. (4.5), (4.6) for $A=0.1$ and $\omega=\frac{2}{\sqrt{3}}+\left(\frac{1}{3 \sqrt{3}}+\right.$ $0.2) A=1.1939$ for initial conditions $x_{1}=x_{2}=0.3$ obtained by numerical integration. This system lies to the right of the resonance tongue in figure 4.3. Note that here a motion which starts near the equilibrium $\left(\frac{1}{3}, \frac{1}{3}\right)$ remains close to it. 


\section{CHAPTER 5}

\section{RPS MODEL WITH PERIODIC TIME-VARYING PAYOFFS - THE \\ GENERAL CASE}

We now return to the more general case where our payoff matrix is,

$$
\begin{gathered}
R \\
P \\
P
\end{gathered}\left(\begin{array}{ccc}
P & S \\
0 & -1+A_{1} \cos \omega t & 1+A_{2} \cos \omega t \\
1+A_{3} \cos \omega t & 0 & -1+A_{4} \cos \omega t \\
-1+A_{5} \cos \omega t & 1+A_{6} \cos \omega t & 0
\end{array}\right)
$$

To help with the analysis, we would like to consider values of $A_{i}$ under which the equilibrium at $\left(\frac{1}{3}, \frac{1}{3}\right)$ is preserved under periodic forcing. From eqs. (4.2) and (4.3), this will require that $G_{1}$ and $G_{2}$ vanish at $x_{1}=x_{2}=\frac{1}{3}$. From that condition, the following relationship arises between the $A_{i}$ coefficients:

$$
\begin{aligned}
& A_{1}=A_{6}+A_{5}-A_{2} \\
& A_{3}=A_{6}+A_{5}-A_{4}
\end{aligned}
$$

In the previous chapter we showed that for a specific case where $A_{1}=-A_{2}=A$ and $A_{3}=A_{4}=A_{5}=A_{6}=0$ the interior equilibrium point changed stability for resonant values of the parameters $\omega$ and $A$. Using perturbation theory, we were able to detect tongues of instability in the parameter space as well as describe the nonlinear behavior in the different regions of the tongues. In this chapter, we seek to investigate the existence of such tongues for the more general case. 


\subsection{Subharmonic Resonance}

We begin by investigating the linear stability of the interior equilibrium point. Recall that the replicator equations for the general model are

$$
\begin{aligned}
& \dot{x_{1}}=x_{1}\left(1-2 x_{2}-x_{1}\right)+x_{1} G_{1} \cos \omega t \\
& \dot{x_{2}}=x_{2}\left(x_{2}+2 x_{1}-1\right)+x_{2} G_{2} \cos \omega t
\end{aligned}
$$

where

$$
\begin{aligned}
& G_{1}=A_{2}\left(1-x_{1}-x_{2}\right)+x_{2}\left[A_{1}-x_{1}\left(A_{1}+A_{3}\right)\right]+F \\
& G_{2}=A_{4}\left(1-x_{1}-x_{2}\right)+x_{1}\left[A_{3}-x_{2}\left(A_{1}+A_{3}\right)\right]+F
\end{aligned}
$$

and

$$
F=\left(x_{1}+x_{2}-1\right)\left[x_{1}\left(A_{2}+A_{5}\right)+x_{2}\left(A_{4}+A_{6}\right)\right]
$$

When we apply the conditions in eqs. (5.2) and (5.3), the governing differential equations become:

$$
\begin{aligned}
\dot{x_{1}}= & x_{1}\left(\left(A_{6}\left(x_{2}^{2}-x_{1} x_{2}\right)+A_{5}\left(x_{2}\left(1-x_{1}\right)+x_{1}^{2}-x_{1}\right)+A_{4}\left(x_{2}^{2}+x_{2}\left(2 x_{1}-1\right)\right)\right.\right. \\
& \left.\left.+A_{2}\left(x_{2}\left(2 x_{1}-2\right)+x_{1}^{2}-2 x_{1}+1\right)\right) \cos \omega t-2 x_{2}-x_{1}+1\right) \\
\dot{x_{2}}= & x_{2}\left(\left(A_{6}\left(x_{2}^{2}-\left(x_{1}+1\right) x_{2}+x_{1}\right)+A_{5}\left(x_{1}^{2}-x_{1} x_{2}\right)+A_{4}\left(x_{2}^{2}+\left(2 x_{1}-2\right) x_{2}+1-2 x_{1}\right)\right.\right. \\
& \left.\left.+A_{2}\left(2 x_{1} x_{2}+x_{1}^{2}-x_{1}\right)\right) \cos \omega t+x_{2}+2 x_{1}-1\right)
\end{aligned}
$$

First we move the interior equilibrium point to the origin for convenience.

$$
x_{1}=x+\frac{1}{3}, \quad x_{2}=y+\frac{1}{3}
$$

Then substitute eqs. (5.11) into (5.9) and (5.10).

$$
\begin{aligned}
\dot{x}= & \frac{1}{9}\left[\operatorname { c o s } \omega t \left\{\left((9 x+3) y^{2}+\left(1-9 x^{2}\right) y-3 x^{2}-x\right) A_{6}\right.\right. \\
& +\left(\left(-9 x^{2}+3 x+2\right) y+9 x^{3}-3 x^{2}-2 x\right) A_{5}
\end{aligned}
$$




$$
\begin{aligned}
& +\left((9 x+3) y^{2}+\left(18 x^{2}+9 x+1\right) y+6 x^{2}+2 x\right) A_{4} \\
& \left.+\left(\left(18 x^{2}-6 x-4\right) y+9 x^{3}-3 x^{2}-2 x\right) A_{2}\right\} \\
& \left.+y(-18 x-6)-9 x^{2}-3 x\right] \\
\dot{y}= & \frac{1}{9}\left[\operatorname { c o s } \omega t \left\{\left(9 y^{3}+(-9 x-3) y^{2}+(3 x-2 y)+2 x\right) A_{6}\right.\right. \\
& +\left((-9 x-3) y^{2}+\left(9 x^{2}-\right) y+3 x^{2}+x\right) A_{5} \\
& +\left(9 y^{3}+(18 x-3) y^{2}+(-6 x-2 y)-4 x\right) A_{4} \\
& \left.+\left((18 x+6) y^{2}+\left(9 x^{2}+9 x+2\right) y+3 x^{2}+x\right) A_{2}\right\} \\
& \left.+9 y^{2}+y(18 x+3)+6 x\right]
\end{aligned}
$$

For a linear stability analysis, we linearize (5.12) and (5.13).

$$
\begin{aligned}
& \dot{x}=\frac{\left((y-x) A_{6}+(2 y-2 x) A_{5}+(y+2 x) A_{4}+(-4 y-2 x) A_{2} x\right) \cos \omega t-6 y-3 x}{9} \\
& \dot{y}=\frac{\left((2 x-2 y) A_{6}+(x-y) A_{5}+(-2 y-4 x) A_{4}+(2 y+x) A_{2}\right) \cos \omega t+3 y+6 x}{9}(5.15)
\end{aligned}
$$

Now we transform this system of first-order ODEs into a second-order ODE for convenience in eliminating secular terms in the upcoming perturbation method. We find

$$
f_{1} \ddot{x}+f_{2} \dot{x}+f_{3} x=0
$$

where

$$
\begin{aligned}
f_{1}= & -9\left(\left(A_{6}+2 A_{5}+A_{4}-4 A_{2}\right) \cos \omega t-6\right) \\
f_{2}= & 18\left(A_{6}+A_{5}\right) \cos \omega t-9 \omega\left(A_{6}+2 A_{5}+A_{4}-4 A_{2}\right) \sin \omega t \\
& -3\left(A_{6}+A_{5}\right)\left(A_{6}+2 A_{5}+A_{4}-4 A_{2}\right) \cos ^{2} \omega t \\
f_{3}= & 18+3\left(A_{6}-4 A_{5}-5 A_{4}+8 A_{2}\right) \cos \omega t-9 \omega\left(A_{6}+2 A_{5}-A_{4}\right) \sin \omega t \\
& +\left(-A_{6}^{2}+\left(-A_{5}+A_{4}+8 A_{2}\right) A_{6}+2 A_{5}^{2}+\left(11 A_{4}-8 A_{2}\right) A_{5}+2 A_{4}^{2}-16 A_{2} A_{4}+8 A_{2}^{2}\right) \cos ^{2} \omega t \\
& -\left(A_{6}+2 A_{5}+A_{4}-4 A_{2}\right)\left(A_{2} A_{6}+A_{4} A_{5}-A_{2} A_{4}\right) \cos ^{3} \omega t
\end{aligned}
$$


We may now use a perturbation method to determine the stability of the interior equilibrium, now located at the origin, under the assumption of small forcing amplitudes. To use the perturbation method we make a change of variables $\tau=\omega t$ and denote' as a derivative with respect to $\tau$. We also write $A_{i} \rightarrow \epsilon A_{i}$. If we neglect terms of $O\left(\epsilon^{2}\right)$, this gives:

$$
g_{1} x^{\prime \prime}+g_{2} x^{\prime}+g_{3} x=O\left(\epsilon^{2}\right)
$$

where

$$
\begin{aligned}
& g_{1}=54 \omega^{2}-9 \omega^{2} \epsilon\left(A_{6}+2 A_{5}+A_{4}-4 A_{2}\right) \cos \tau \\
& g_{2}=18 \omega \epsilon\left(A_{6}+A_{5}\right) \cos \tau-9 \omega^{2} \epsilon\left(A_{6}+2 A_{5}+A_{4}-4 A_{2}\right) \sin \tau \\
& g_{3}=18+3 \epsilon\left(A_{6}-4 A_{5}-5 A_{4}+8 A_{2}\right) \cos \tau-9 \epsilon \omega\left(A_{6}+2 A_{5}-A_{4}\right) \sin \tau
\end{aligned}
$$

To begin with, we determine the resonant value of $\omega$ at $O(\epsilon)$ by setting

$$
x=x_{0}+\epsilon x_{1}+O\left(\epsilon^{2}\right)
$$

Substituting (5.24) into (5.20) and collecting terms gives:

$$
\begin{aligned}
& x_{0}^{\prime \prime}+\frac{x_{0}}{3 \omega^{2}}=0 \\
& x_{1}^{\prime \prime}+\frac{x_{1}}{3 \omega^{2}}=H_{1} x_{0}^{\prime \prime}+H_{2} x_{0}^{\prime}+H_{3} x_{0}
\end{aligned}
$$

where

$$
\begin{aligned}
& H_{1}=\frac{\left(A_{6}+2 A_{5}+A_{4}-4 A_{2}\right) \cos \tau}{6} \\
& H_{2}=\frac{\omega\left(A_{6}+2 A_{5}+A_{4}-4 A_{2}\right) \sin \tau+\left(-2 A_{6}-2 A_{5}\right) \cos \tau}{6 \omega} \\
& H_{3}=\frac{\omega\left(3 A_{6}+6 A_{5}-3 A_{4}\right) \sin \tau+\left(-A_{6}+4 A_{5}+5 A_{4}-8 A_{2}\right) \cos \tau}{18 \omega^{2}}
\end{aligned}
$$

From (5.25), we see that $x_{0}$ will have a solution with frequency $\frac{1}{\sqrt{3} \omega}$ whereupon the right hand side of eq. (5.26) will have terms with frequencies:

$$
1 \pm \frac{1}{\sqrt{3} \omega}
$$


Resonant values of $\omega$ will correspond to forcing frequencies (5.30) which are equal to natural frequencies of the homogeneous $x_{1}$ equation, i.e. to $\frac{1}{\sqrt{3} \omega}$. This gives that

$$
\omega=\frac{2}{\sqrt{3}} \quad \text { (resonance) }
$$

This value of $\omega$ corresponds to the largest resonance tongue. There are an infinitude of smaller tongues which would emerge from the perturbation method if we were to continue it to $O\left(\epsilon^{2}\right)$ and higher. These have been shown [15] to be of the form $\omega_{0}=2 /(n \sqrt{3})$ for $n=2,3, \ldots$

In order to investigate the nature of the dynamical behavior in the neighborhood of the resonance (5.31), we define two time scales $\xi$ and $\eta$

$$
\xi=\tau, \quad \eta=\epsilon \tau
$$

and we consider $x$ to be a function of $\xi$ and $\eta$, whereupon the chain rule gives:

$$
\begin{aligned}
x^{\prime} & =x_{\xi}+\epsilon x_{\eta} \\
x^{\prime \prime} & =x_{\xi \xi}+2 \epsilon x_{\xi \eta}+\epsilon^{2} x_{\eta \eta}
\end{aligned}
$$

We detune $\omega$ off of the resonance (5.31):

$$
\omega=\frac{2}{\sqrt{3}}+k_{1} \epsilon+\cdots
$$

and expand $x=x_{0}+\epsilon x_{1}+\cdots$. Substituting (5.33), (5.34) and these expansions into (5.20) and collecting terms, we obtain:

$$
\begin{aligned}
& x_{0 \xi \xi}+\frac{1}{4} x_{0}=0 \\
& x_{1 \xi \xi}+\frac{1}{4} x_{1}=-2 x_{0 \xi \eta}+h_{1} x_{0 \xi \xi}+h_{2} x_{0 \xi}+h_{3} x_{0}+\frac{\sqrt{3}}{4} k_{1} x_{0}
\end{aligned}
$$

where the functions $h_{i}$ in eq.(5.37) are the same as the functions $H_{i}$ in eq.(5.20) with $\tau$ replaced by $\xi$ and $\omega$ replaced by $\frac{2}{\sqrt{3}}$. 
We take the solution of (5.36) in the form:

$$
x_{0}=a(\eta) \cos \frac{\xi}{2}+b(\eta) \sin \frac{\xi}{2}
$$

We substitute the expression for $x_{0}$ (5.38) into the $x_{1}$ equation (5.37), and remove secular terms, giving the slow flow:

$$
\begin{aligned}
& \frac{\partial a}{\partial \eta}=a\left(\frac{A_{4}-A_{5}}{8 \sqrt{3}}\right)+b\left(-\frac{\sqrt{3}}{4} k_{1}+\frac{A_{2}-A_{6}}{12}+\frac{A_{4}-A_{5}}{24}\right) \\
& \frac{\partial b}{\partial \eta}=-b\left(\frac{A_{4}-A_{5}}{8 \sqrt{3}}\right)+a\left(\frac{\sqrt{3}}{4} k_{1}+\frac{A_{2}-A_{6}}{12}+\frac{A_{4}-A_{5}}{24}\right)
\end{aligned}
$$

Eqs. (5.39), (5.40) are a constant coefficient linear system with the following eigenvalues:

$$
\pm \frac{1}{12} \sqrt{-27 k_{1}^{2}+\left(A_{2}-A_{6}\right)^{2}+\left(A_{4}-A_{5}\right)^{2}+\left(A_{2}-A_{6}\right)\left(A_{4}-A_{5}\right)}
$$

For given parameters $A_{2}, A_{4}, A_{5}, A_{6}$, the equilibrium point $a=b=0$ will be either unstable (exponential growth) or stable (quasiperiodic motion) depending respectively on whether the eigenvalues (5.41) are real or imaginary. The transition between stable and unstable will correspond to zero eigenvalues, given by the condition:

$$
27 k_{1}^{2}=\left(A_{2}-A_{6}\right)^{2}+\left(A_{4}-A_{5}\right)^{2}+\left(A_{2}-A_{6}\right)\left(A_{4}-A_{5}\right)
$$

Eq. (5.42) will yield two values of $k_{1}$, let's call them $k_{1}= \pm Q$, which from eq. (5.35) plot as two straight lines in the $\omega-\epsilon$ plane, representing the boundaries of the 2:1 subharmonic resonance tongue, see figure 5.1. Inside this tongue the equilibrium is unstable due to parametric resonance:

$$
\omega=\frac{2}{\sqrt{3}} \pm Q \epsilon, \quad Q=\frac{\sqrt{\left(A_{2}-A_{6}\right)^{2}+\left(A_{4}-A_{5}\right)^{2}+\left(A_{2}-A_{6}\right)\left(A_{4}-A_{5}\right)}}{\sqrt{27}}
$$




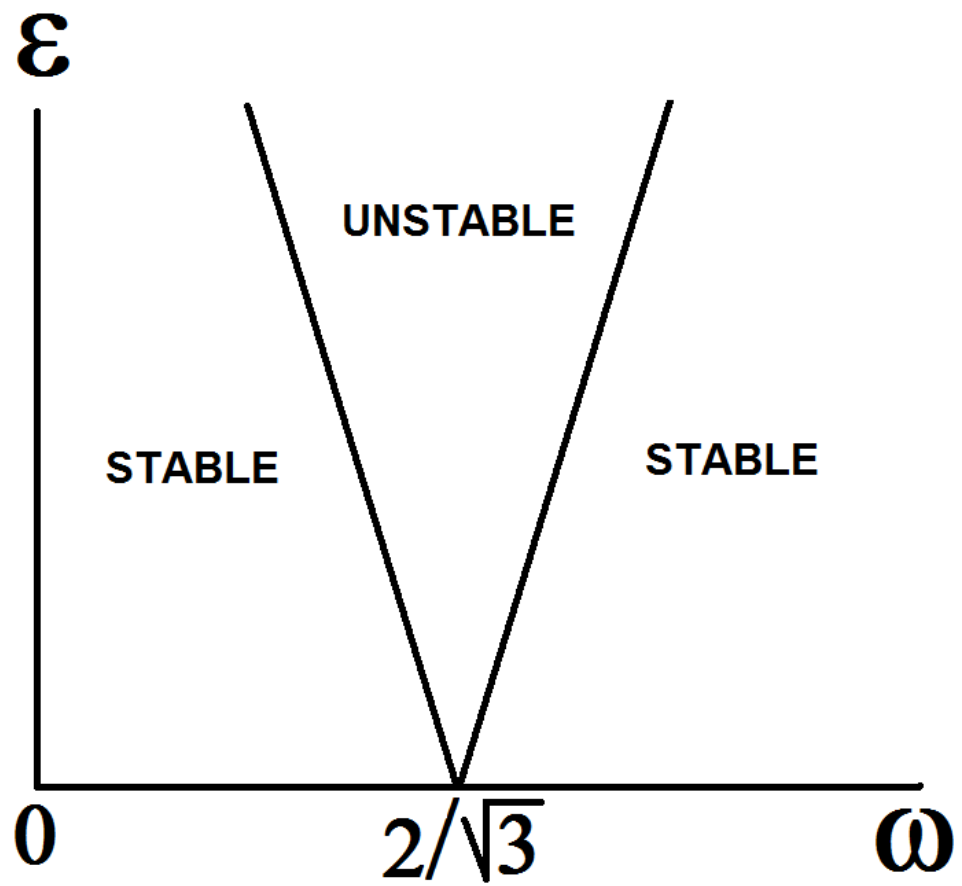

Figure 5.1: 2:1 subharmonic resonance tongue, eq.(5.43). The RPS equilibrium point at $x_{1}=x_{2}=\frac{1}{3}$ is linearly unstable for parameters inside the tongue. The presence of nonlinearities detunes the resonance and prevents unbounded motions which are predicted by the linear stability analysis.

\subsection{Disappearing Tongue}

In the special case that $A_{2}=A_{6}$ and $A_{4}=A_{5}$, we see from eq. (5.43) that $Q=0$ and the tongue has closed up, at least to $O(\epsilon)$. For these parameter values we have from eqs. (5.2), (5.3):

$$
A_{1}=A_{4}=A_{5} \equiv \alpha \epsilon, \quad A_{2}=A_{3}=A_{6} \equiv \beta \epsilon
$$


so that the payoff matrix (4.1) becomes:

$$
P\left(\begin{array}{ccc}
R & P & S \\
0 & -1+\alpha \epsilon \cos \omega t & 1+\beta \epsilon \cos \omega t \\
1+\beta \epsilon \cos \omega t & 0 & -1+\alpha \epsilon \cos \omega t \\
-1+\alpha \epsilon \cos \omega t & 1+\beta \epsilon \cos \omega t & 0
\end{array}\right)
$$

where $\omega=2 / \sqrt{3}$, and the linearized differential eqs. (5.14), (5.15) become:

$$
\begin{aligned}
& \dot{x}=\frac{(y \alpha \epsilon-(x+y) \beta \epsilon) \cos \omega t-2 y-x}{3} \\
& \dot{y}=\frac{(-(x+y) \alpha \epsilon+x \beta \epsilon) \cos \omega t+y+2 x}{3}
\end{aligned}
$$

From Floquet theory [20],[15] we know that on the transition curves which define the two sides of the tongue, i.e. which separate regions of stability from regions of instability, there exists a periodic solution having frequency $\omega / 2$ (a "subharmonic"). To prove that the tongue has truly disappeared (rather than approximately so as in perturbation theory), we must show that there COEXIST two linearly independent solutions having frequency $\omega / 2$. To make this easier to consider, define a new subharmonic time scale $T=(\omega / 2) t=t / \sqrt{3}$. Then eqs. (5.46), (5.47) become:

$$
\begin{aligned}
& \frac{1}{\sqrt{3}} \frac{d x}{d T}=\frac{(y \alpha \epsilon-(x+y) \beta \epsilon) \cos 2 T-2 y-x}{3} \\
& \frac{1}{\sqrt{3}} \frac{d y}{d T}=\frac{(-(x+y) \alpha \epsilon+x \beta \epsilon) \cos 2 T+y+2 x}{3}
\end{aligned}
$$

Here we must show that there exists two linearly independent solutions with frequency 1 in $T$. For example, when $\alpha=\beta=0$, there are two linearly independent solutions with frequency 1:

$$
x=\sqrt{3} \cos T-\sin T, \quad y=2 \sin T
$$

and 


$$
x=-2 \sin T, \quad y=\sqrt{3} \cos T+\sin T
$$

That is, we are forcing the system at twice its natural frequency. The idea here is that there normally exists a solution of frequency 1 on each transition curve. In order to show that there is no tongue, we have to show that the two transition curves are coincident. In fact we claim that the two transition curves correspond to $k_{1}=Q=0$, that is, to a single vertical line in the $\omega-\epsilon$ plane, going through the point $\omega=2 / \sqrt{3}, \epsilon=0$. Eqs. (5.48), (5.49) correspond to such a vertical line, and so we want to show that there are two linearly independent solutions to these equations.

Numerical simulations of eqs. (5.48), (5.49) have shown that this result is valid to all orders of $\epsilon$, i.e., eqs. (5.48), (5.49) exhibit a frequency 1 solution for all nontrivial initial conditions, regardless of the values of $\alpha, \beta$ or $\epsilon$. That is, the tongue really does close up and the instability disappears. Moreover, numerical evidence shows that all the other tongues in the $\epsilon-\omega$ plane (which emanate from points on the $\omega$-axis at $\omega=2 /(n \sqrt{3})$, see [15]) also close up and disappear.

We supplement these numerical results with the following THEOREM: All nontrivial solutions to eqs. (5.48), (5.49) are periodic with frequency 1.

Proof: We assume a solution to eqs. (5.46), (5.47) in the form ("variation of parameters"):

$$
x=u(\sqrt{3} \cos T-\sin T)+v(-2 \sin T)
$$




$$
y=u(2 \sin T)+v(\sqrt{3} \cos T+\sin T)
$$

where $u$ and $v$ are functions of $T$ to be found. Note that $(u=1, v=0)$ gives (5.50), while $(u=0, v=1)$ gives (5.51). Substituting (5.52), (5.53) into (5.48), (5.49) gives the following eqs. on $u$ and $v$ :

$$
\begin{aligned}
& \sqrt{3} \frac{d u}{d T}=\epsilon \cos 2 T(-\beta u+(\alpha-\beta) v) \\
& \sqrt{3} \frac{d v}{d T}=\epsilon \cos 2 T((\beta-\alpha) u-\alpha v)
\end{aligned}
$$

Next we define new time variable $z$ :

$$
d z=\frac{\epsilon \cos 2 T}{\sqrt{3}} d T \quad \Rightarrow \quad z=\frac{\epsilon \sin 2 T}{2 \sqrt{3}}
$$

which gives the following constant coefficient linear system on $u, v$ :

$$
\frac{d}{d z}\left[\begin{array}{l}
u \\
v
\end{array}\right]=\left[\begin{array}{cc}
-\beta & \alpha-\beta \\
\beta-\alpha & -\alpha
\end{array}\right]\left[\begin{array}{l}
u \\
v
\end{array}\right]
$$

The matrix in (5.57) has eigenvalues:

$$
\lambda=-\left(\frac{\alpha+\beta}{2}\right) \pm i \frac{\sqrt{3}}{2}(\beta-\alpha)
$$

Thus the general solution to (5.57) involves a linear combination of terms of the form

$$
\exp \left(\frac{\alpha+\beta}{2} z\right)\left[\begin{array}{c}
\sin \\
\cos
\end{array}\right] \frac{\sqrt{3}}{2}(\beta-\alpha) z
$$

Therefore since $z$ is a $\pi$-periodic function of $T$, we see that $u$ and $v$ also have period $\pi$ in time $T$. Then from (5.52), (5.53), it follows that $x$ and $y$ have period $2 \pi$ in $T$, since the product of a $\pi$-periodic function and a $2 \pi$-periodic function has period $2 \pi$. Q.E.D. 


\subsection{Conclusion}

From a dynamical systems point of view, we may summarize our findings as follows: The original RPS system, with payoff matrix (3.2) and no forcing, exhibits an equilibrium at $\left(\frac{1}{3}, \frac{1}{3}\right)$ which is stable. With the addition of forcing, there will generally be a 2:1 subharmonic resonance region in parameter space in which the equilibrium becomes unstable. In this chapter we have shown that this tongue may be absent or very small if the forcing parameters are chosen appropriately.

In the case that the equilibrium is linearly unstable, the presence of nonlinearities detunes the resonance (because the frequency of the motion changes as the amplitude increases) and prevents the unbounded motions which are predicted by the linear stability analysis. The resulting unstable motion is either quasiperiodic or chaotic as described in Chapter 4.

From a biological and social point of view, the presence of periodic forcing in RPS can lead to quasiperiodic or chaotic oscillations, such as those observed in the range of biological and social applications. Seemingly stochastic fluctuations in strategy frequencies need not necessarily arise from a stochastic process, as we have shown in earlier work in the previous chapter. Now we see that depending on the choice of forcing parameters, it is possible to reduce or even eliminate quasiperiodic motion. Thus, if one was designing an organization, community or political system where stability was desired, this effect could be achieved by properly tuning the degree of periodic forcing. A similar logic applies to biological systems. If the forcing coefficients were themselves subject to natural selection, evolution might favor coefficients that eliminate the tongue 
and result in stable population frequencies. 


\section{CHAPTER 6}

\section{DISAPPERANCE OF RESONACE TONGUES}

In the previous chapter, we were able to show that for certain parameters the largest resonance tongue at $\omega=\frac{2}{\sqrt{3}}$ completely disappears and there coexists two linearly independent solutions that are both $2 \pi$ periodic. In this chapter we consider a more general system of ODEs for which the previous system,eqs. (5.48), (5.49), is a subset of. Here we aim to prove that the resonance tongues disappear in the general system given certain parameters and that the system in the previous chapters meets those conditions. We begin by providing a motivating example that captures the result we obtained in our previous chapter. Here we use perturbation methods to find approximate solutions which will suggest that for some special parameters there is no region of instability. However, since these are only approximate solutions we cannot be certain the tongue has indeed disappeared. The system with periodic coefficients that we encountered in eqs. (5.48) and (5.49) can be written in the form:

$$
\begin{aligned}
& \sqrt{3} \delta \frac{d x}{d t}=(-1+2(\mu-1) \epsilon \cos 2 t) x+(-2+(2+\mu) \epsilon \cos 2 t) y \\
& \sqrt{3} \delta \frac{d y}{d t}=(2+(1-4 \mu) \epsilon \cos 2 t) x+(1-(1+2 \mu) \epsilon \cos 2 t) y
\end{aligned}
$$

where $\delta, \mu$ and $\epsilon$ are parameters. For fixed $\mu$, we picture the $\delta-\epsilon$ plane and desire to know for which regions all solutions to (6.1) and (6.2) are bounded (in which case these equations are said to be stable, and for which regions unbounded solutions exist (unstable). We are interested in the limit in which $\epsilon \rightarrow 0$ so we

expand $\delta=1+k \epsilon+O\left(\epsilon^{2}\right)$. As in the case of Mathieu's equation [14] [20], there is, for typical values of $\mu$, a tongue of instability emanating from the point $\delta=1$, $\epsilon=0$ on the $\delta$-axis. This instability is caused by a 2:1 subharmonic resonance. 
This can be seen by noting that for $\epsilon=0$, eqs. (6.1) and (6.2) take the form

$$
\sqrt{3} \frac{d}{d t}\left(\begin{array}{l}
x \\
y
\end{array}\right)=\left(\begin{array}{cc}
-1 & -2 \\
2 & 1
\end{array}\right)\left(\begin{array}{l}
x \\
y
\end{array}\right)
$$

Eqs. (6.3) have the general solution:

$$
\left(\begin{array}{l}
x \\
y
\end{array}\right)=A\left(\begin{array}{c}
\sqrt{3} \cos t-\sin t \\
2 \sin t
\end{array}\right)+B\left(\begin{array}{c}
-2 \sin t \\
\sqrt{3} \cos t+\sin t
\end{array}\right)
$$

where $A$ and $B$ are arbitrary constants. Note that the forcing function in eqs. (6.1) and (6.2), $\cos 2 t$, has twice the frequency of the solution (6.4) of the unforced system (6.3), which is the source of the 2:1 resonance. We seek an approximate solution to eqs.(6.1) and (6.2) valid for small $\epsilon$ by using the two variable expansion method [14], also known as the method of multiple scales [12]. We replace the independent variable $t$ by two time variables, $\xi=t$ and $\eta=\epsilon t$, whereupon eqs. (6.1) and (6.2) become:

$$
\begin{aligned}
& \sqrt{3}\left(1+k \epsilon+O\left(\epsilon^{2}\right)\right)\left(\frac{\partial x}{\partial \xi}+\epsilon \frac{\partial x}{\partial \eta}\right)=(-1+2(\mu-1) \epsilon \cos 2 \xi) x+(-2+(2+\mu) \epsilon \cos 2 \xi 5) \\
& \sqrt{3}\left(1+k \epsilon+O\left(\epsilon^{2}\right)\right)\left(\frac{\partial y}{\partial \xi}+\epsilon \frac{\partial y}{\partial \eta}\right)=(2+(1-4 \mu) \epsilon \cos 2 \xi) x+(1-(1+2 \mu) \epsilon \cos 2 \xi)(6.6)
\end{aligned}
$$

Next we expand $x=x_{0}+\epsilon x_{1}+O\left(\epsilon^{2}\right)$ and $y=y_{0}+\epsilon y_{1}+O\left(\epsilon^{2}\right)$ and collect terms, giving:

$$
\begin{gathered}
\sqrt{3} \frac{\partial x_{0}}{\partial \xi}=-x_{0}-2 y_{0} \\
\sqrt{3} \frac{\partial y_{0}}{\partial \xi}=2 x_{0}+y_{0} \\
\left.\sqrt{3} \frac{\partial x_{1}}{\partial \xi}=-x_{1}-2 y_{1}+2(\mu-1) x_{0} \cos 2 \xi+(2+\mu) y_{0} \cos 2 \xi-\sqrt{3} k \frac{\partial x_{0}}{\partial \xi}-\sqrt{3} \frac{\partial x_{0}}{\partial \eta} \cdot 9\right) \\
\left.\sqrt{3} \frac{\partial y_{1}}{\partial \xi}=2 x_{1}+y_{1}+(1-4 \mu) x_{0} \cos 2 \xi-(1+2 \mu) y_{0} \cos 2 \xi-\sqrt{3} k \frac{\partial y_{0}}{\partial \xi}-\sqrt{3} \frac{\partial y_{0}}{\partial \eta} 10\right)
\end{gathered}
$$


Eqs. (6.7), (6.8) have the solution (cf. eqs. (6.4)):

$$
\left(\begin{array}{l}
x_{0} \\
y_{0}
\end{array}\right)=A(\eta)\left(\begin{array}{c}
\sqrt{3} \cos \xi-\sin \xi \\
2 \sin \xi
\end{array}\right)+B(\eta)\left(\begin{array}{c}
-2 \sin \xi \\
\sqrt{3} \cos \xi+\sin \xi
\end{array}\right)
$$

Next we substitute eqs. (6.11) into eqs. (6.9), (6.10) and trigonometrically reduce the resulting equations, which are of the form:

$$
\begin{aligned}
& \sqrt{3} \frac{\partial x_{1}}{\partial \xi}=-x_{1}-2 y_{1}+M_{1} \cos \xi+M_{2} \sin \xi+N . R . T . \\
& \sqrt{3} \frac{\partial y_{1}}{\partial \xi}=2 x_{1}+y_{1}+M_{3} \cos \xi+M_{4} \sin \xi+N . R . T .
\end{aligned}
$$

where N.R.T. stands for Non-Resonant Terms, and where the coefficients $M_{i}$ are known functions of $A(\eta)$ and $B(\eta)$, omitted here for brevity. For no secular terms in $x_{1}$ and $y_{1}$, the following relationships must hold:

$$
\begin{aligned}
& 2 M_{1}+M_{3}+\sqrt{3} M_{4}=0 \\
& 2 M_{2}-\sqrt{3} M_{3}+M_{4}=0
\end{aligned}
$$

Eqs. (6.14), (6.15) give the following slow flow:

$$
\begin{aligned}
& 2 \sqrt{3} \frac{d A}{d \eta}=(2 k+\mu-1) A+(4 k-\mu+1) B \\
& 2 \sqrt{3} \frac{d B}{d \eta}=(4 k+2 \mu-2) A+(2 k+\mu-1) B
\end{aligned}
$$

Eqs. (6.16), (6.17) are a linear constant coefficient system with eigenvalues

$$
\lambda= \pm \frac{1}{2} \sqrt{(\mu-1)^{2}-4 k^{2}}
$$

The transition between stable and unstable corresponds to $\lambda=0$ :

$$
\lambda=0 \Rightarrow k= \pm \frac{1}{2}(\mu-1)
$$

Since $\delta=1+k \epsilon+O\left(\epsilon^{2}\right)$, we see that the tongue of instability has boundaries given by:

$$
\delta=1 \pm \frac{1}{2}(\mu-1) \epsilon+O\left(\epsilon^{2}\right)
$$


In particular we see that when $\mu=1$, the tongue closes up to $\mathrm{O}\left(\epsilon^{2}\right)$. Numerical simulations confirm that as $\mu \rightarrow 1$ the region of instability begins to narrow. In figure 6.1 we see that the boundaries of the resonance tongues intersect each other. These intersections increase in frequency as $\mu \rightarrow 1$ until eventually the two boundaries become a straight vertical line at $\delta=1$.

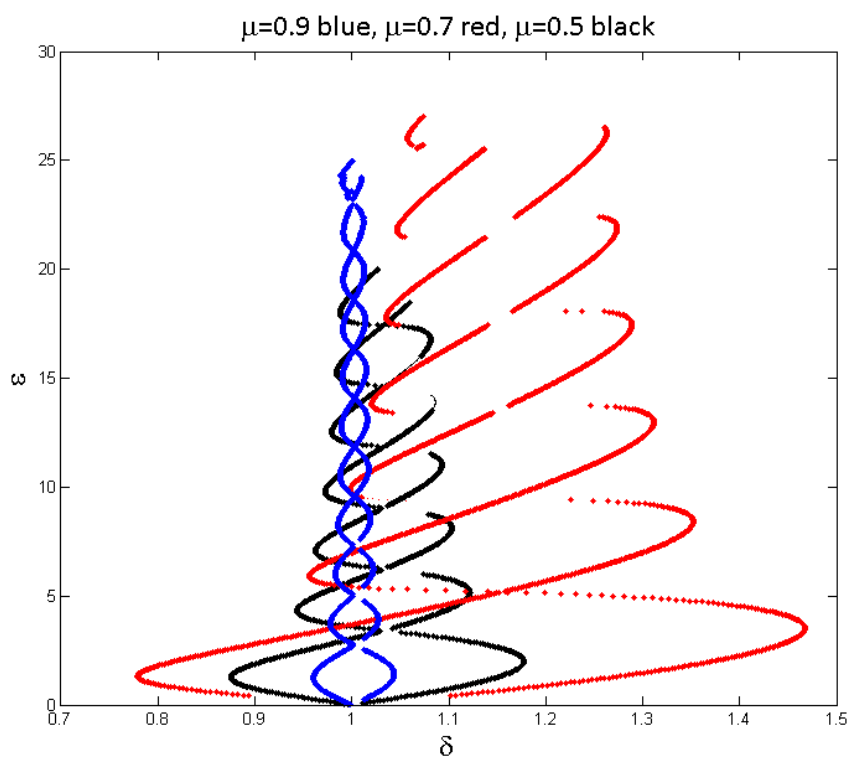

Figure 6.1: The boundaries of the resonance tongue calculated numerically. Red is when $\mu=0.5$, black is when $\mu=0.7$, and blue is when $\mu=0.9$. The boundaries of the tongue intersect multiple times and more frequently as $\mu \rightarrow 1$.

The question is, is this an approximate result, valid only to some order of $\epsilon$, or is it exact, valid to all orders of $\epsilon$ ? 


\subsection{A Theorem}

In this section we generalize the preceding example by considering a system in the following form:

$$
\begin{aligned}
& \frac{d x}{d t}=a_{1}(t) x+a_{2}(t) y \\
& \frac{d y}{d t}=a_{3}(t) x+a_{4}(t) y
\end{aligned}
$$

where

$$
a_{i}(t)=P_{i}+\epsilon Q_{i} \cos 2 t
$$

We assume that when $\epsilon=0$, the system (6.21), (6.22) has a pair of linearly independent solutions of period $2 \pi$ (cf. eqs. (6.4)), which we write in the form:

$$
\begin{aligned}
& x=A F_{1}(t)+B F_{2}(t) \\
& y=A G_{1}(t)+B G_{2}(t)
\end{aligned}
$$

where $A$ and $B$ are arbitrary constants.

The goal is then to find conditions on the coefficients $P_{i}$ and $Q_{i}$ such that eqs. (6.21), (6.22) have two linearly independent solutions of period $2 \pi$ for all $\epsilon>0$.The coexistence of these two solutions for all $\epsilon>0$ means that the associated 2:1 resonance tongue has closed up, both boundaries being coincident. (As we saw in the foregoing example, in general each boundary of the tongue possesses a period $2 \pi$ solution. If the system (6.21), (6.22) possesses two linearly independent period $2 \pi$ solutions, then both tongue boundaries are coincident, and the tongue has closed up.) 
When $\epsilon=0$, eqs.(6.21),(6.22) become

$$
\frac{d}{d t}\left(\begin{array}{l}
x \\
y
\end{array}\right)=\left(\begin{array}{cc}
P_{1} & P_{2} \\
P_{3} & P_{4}
\end{array}\right)\left(\begin{array}{l}
x \\
y
\end{array}\right)
$$

For eqs.(6.26) to exhibit two linearly independent solutions of period $2 \pi$, its eigenvalues must be $\pm i$. This requires that the trace of the matrix in (6.26) be zero, and its determinant be unity, giving:

$$
\begin{array}{r}
P_{4}=-P_{1} \\
P_{3}=\frac{-1-P_{1}^{2}}{P_{2}}
\end{array}
$$

Then without loss of generality we may take the two linearly independent period $2 \pi$ solutions in $(6.24),(6.25)$ to be:

$$
F_{1}(t)=\sin t, \quad G_{1}(t)=v_{1} \sin t+v_{2} \cos t
$$

and

$$
G_{2}(t)=\sin t, \quad F_{2}(t)=v_{3} \sin t+v_{4} \cos t
$$

where the $v_{i}$ coefficients may be found by substituting (6.29), (6.30) into the $\epsilon=0$ equations (6.26), giving:

$$
\begin{aligned}
& v_{1}=-\frac{P_{1}}{P_{2}} \\
& v_{2}=\frac{1}{P_{2}} \\
& v_{3}=-\frac{P_{1} P_{2}}{1+P_{1}^{2}} \\
& v_{4}=-\frac{P_{2}}{1+P_{1}^{2}}
\end{aligned}
$$

Having characterized the solution of eqs.(6.21), (6.22) for $\epsilon=0$, we now go after the solution for $\epsilon>0$. 
We posit a solution for $\epsilon>0$ using variation of parameters (cf. eqs.(6.24),(6.25)):

$$
\begin{aligned}
& x=u(t) F_{1}(t)+v(t) F_{2}(t) \\
& y=u(t) G_{1}(t)+v(t) G_{2}(t)
\end{aligned}
$$

where $u(t)$ and $v(t)$ are unknown functions to be found. Substituting (6.35), (6.36) into (6.21), (6.22), and solving for $d u / d t$ and $d v / d t$, we get

$$
\begin{aligned}
& \frac{d u}{d t}=\epsilon \cos 2 t\left[H_{1} \sin ^{2} t+H_{2} \sin t \cos t+H_{3}\right] \\
& \frac{d v}{d t}=\epsilon \cos 2 t\left[H_{4} \sin ^{2} t+H_{5} \sin t \cos t+H_{6}\right]
\end{aligned}
$$

where $H_{i}=H_{i}\left(P_{1}, P_{2}, Q_{i}, u, v\right)$ are known functions, too long to list here.

Motivated by a desire to find conditions which guarantee a pair of linearly independent solutions to eqs. (6.21), (6.22), we set $H_{1}=H_{2}=H_{4}=H_{5}=0$, which requires

$$
\begin{aligned}
& Q_{2}=-\frac{P_{2}}{2 P_{1}}\left(Q_{4}-Q_{1}\right) \\
& Q_{3}=\frac{\left(1+P_{1}^{2}\right)}{2 P_{1} P_{2}}\left(Q_{4}-Q_{1}\right)
\end{aligned}
$$

Assuming that conditions (6.39), (6.40) are fulfilled, eqs.(6.37),(6.38) become:

$$
\begin{aligned}
& \frac{d u}{d t}=\epsilon \cos 2 t\left[Q_{4} u-\frac{P_{2}}{2 P_{1}}\left(Q_{4}-Q_{1}\right) v\right] \\
& \frac{d v}{d t}=\epsilon \cos 2 t\left[\frac{1+P_{1}^{2}}{2 P_{1} P_{2}}\left(Q_{4}-Q_{1}\right) u+Q_{1} v\right]
\end{aligned}
$$

Next we reparameterize $t$ by using a new time scale $\tau$ defined as:

$$
\tau=\frac{1}{2} \sin 2 t
$$


which transforms (6.41), (6.42) into a system with constant coefficients:

$$
\begin{aligned}
& \frac{d u}{d \tau}=\epsilon\left[Q_{4} u-\frac{P_{2}}{2 P_{1}}\left(Q_{4}-Q_{1}\right) v\right] \\
& \frac{d v}{d \tau}=\epsilon\left[\frac{1+P_{1}^{2}}{2 P_{1} P_{2}}\left(Q_{4}-Q_{1}\right) u+Q_{1} v\right]
\end{aligned}
$$

Although the solution of eqs. (6.44), (6.45) depends on the numerical values of the coefficients $P_{1}, P_{2}, Q_{1}, Q_{4}$, it will in general consist of sinusoidal and or exponential functions of $\tau$, and since $\tau$ is $\pi$-periodic in $t$ by eq.(6.43), $u(t)$ and $v(t)$, the solution functions of (6.44), (6.45), will be $\pi$-periodic in $t$. Furthermore, we note by eqs. (6.35), (6.36), that $x(t)$ and $y(t)$, the solution functions of eqs. (6.21), (6.22), are composed of terms which are the product of $\pi$-periodic functions (i.e. $u(t)$ and $v(t))$ and $2 \pi$-periodic functions (i.e. $F_{i}(t)$ and $\left.G_{i}(t)\right)$. Now since the product of a $\pi$-periodic function and a $2 \pi$-periodic function has period $2 \pi$, we see that all solutions of eqs. (6.21), (6.22) are $2 \pi$ periodic.

We have therefore proved the following

THEOREM: All nontrivial solutions of eqs. (6.21), (6.22) will have period $2 \pi$ if eqs. (6.27), (6.28), (6.39), (6.40) are satisfied.

\subsection{Application}

In this section we apply the theorem of the previous section to the example presented earlier in this chapter. Eqs. (6.1), (6.2), when expressed in the form of 
eqs. (6.21), (6.22) yield the following values for the coefficients $P_{i}$ and $Q_{i}$ :

$$
\begin{aligned}
& P_{1}=-\frac{1}{\sqrt{3}} \\
& P_{2}=-\frac{2}{\sqrt{3}} \\
& P_{3}=\frac{2}{\sqrt{3}} \\
& P_{4}=\frac{1}{\sqrt{3}} \\
& Q_{1}=\frac{-2+2 \mu}{\sqrt{3}} \\
& Q_{2}=\frac{2+\mu}{\sqrt{3}} \\
& Q_{3}=\frac{1-4 \mu}{\sqrt{3}} \\
& Q_{4}=-\frac{1+2 \mu}{\sqrt{3}}
\end{aligned}
$$

Inspection shows that these values for $P_{i}$ and $Q_{i}$ satisfy eqs .(6.27), (6.28), (6.39), (6.40) in the case that $\mu=1$. Therefore we may conclude by the foregoing theorem that the closing of the tongue at $\mu=1$ is an exact result and is valid to all orders of $\epsilon$.

\subsection{Conclusion}

The perturbation methods used to find approximate solutions to eqs. (6.1), (6.2) suggest that for the special value of $\mu=1$ there exists no region of instability. Meaning that the resonance tongue has disappeared. The results, however, are only approximate and using this method we cannot account for all of the higher order terms. Therefore we set out to prove the disappearance of the resonance tongue in a different manner. We consider a more general class of differential equations, seen in eqs. (6.21)-(6.25), for which our original problem is an exam- 
ple of. We chose our parameters carefully so that solutions are guaranteed to be comprised of two bounded and linearly independent functions of period $2 \pi$. This leads to the choice of parameters found in eqs. (6.27), (6.28), (6.39), (6.40). We then show that our original problem satisfies those conditions and, hence, does not exhibit a resonance tongue. 


\section{CHAPTER 7}

\section{CONCLUSION}

We began our investigation of the RPS model with time-varying coefficients by considering a simple case where there were only two parameters, $A$ and $\omega$. While the standard RPS model is characterized by neutral cycles surrounding the interior equilibrium point, our model showed quasiperiodic behavior and evidence of a change in stability in the interior equilibrium point. We then sought to find for which values of $A$ and $\omega$ the interior equilibrium point exhibited linear resonance. Through perturbation analysis we found that there existed tongues of instability in the parameter space and that inside of these tongues the interior equilibrium point was unstable. Using a multiple-scale perturbation method we were able to derive equations for the slow flow of the system and see the difference in behavior of the model both inside and outside of the resonant tongue. We found the the tongue was not symmetric, meaning the behavior to the left of the tongue was qualitatively different then the behavior to the right of the tongue. The results from the slow flow are valid for small values of $A$ and are supported by numerically generated Poincaré maps. For large values of $A$ we see the appearance of chaos which is associated with KAM theory.

Following the case with only two parameters, we investigated the dynamics of our time-varying RPS model for all parameter values for which the interior equilibrium point remained. We found that, in general, our model has a 2:1 subharmonic resonance region in parameter space where the interior equilibrium becomes unstable. Using multiple time scales and perturbation methods we were able to determine to linear order the boundaries in parameter space for 
the instability region. We were surprised to find that there existed values for $A_{i}$ in which our linear approximation of the boundary would disappear. Numerical integration tests also showed that the instability region had closed. In order to show that the instability region had indeed disappeared and was not simply too small to find numerically we sought to prove the result analytically. To prove that the resonant tongue had disappeared for those parameter values we needed to show that there coexisted two linearly independent solutions having frequency $\omega / 2$. Using variation of parameters, we were able to postulate a form of the solution to a scaled version of our dynamical system. We proved that the solution we postulated did in fact solve our differential equations and that it was composed of two linearly independent terms having frequency $\omega / 2$.

Next we expanded our proof of disappearing tongues of instability to apply to a broader set of differential equations of which our previous system belonged. Again, using variation of parameters we were able to find conditions on our proposed solutions for which there existed two linearly independent solutions that were $2 \pi$ periodic. 


\section{BIBLIOGRAPHY}

[1] V.I. Arnold and A. Avez. Ergodic Problems of Classical Mechanics. Benjamin, New York, 1968.

[2] Robert M. Axelrod. The Evolution of Cooperation. Basic books, 2006.

[3] Carl M. Bender and Steven A. Orszag. Advance Mathematical Methods for Scientists and Engineers: Asymptotic Methods and Perturbation Theory. Springer, 1999.

[4] Ross Cressman. Evolutionary Dynamics and Extensive Form Games. The MIT Press, 2003.

[5] John Guckenheimer and Philip Holmes. Nonlinear Oscillations, Dynamical Systems, and Bifurcations of Vector Fields. Springer, New York, 1983.

[6] Josef Hofbauer and Karl Signmund. Evolutionary Games and Population Dynamics. Cambridge University Press, 1998.

[7] Benjamin Kerr, Margaret A. Riley, Marcus W. Feldman, and Brendan J. M. Bohannan. Local dispersal promotes biodiversity in a real-life game of rock-paper-scissors. Nature, 2002.

[8] Benjamin C. Kirkup and Margaret A. Riley. Antibiotic-mediated antagonism leads to a bacterial game of rock-paper-scissors in vivo. Nature, 2004.

[9] Yuri A. Kuznetsov. Elements of Applied Bifurcation Theory. Springer, New York, 2004.

[10] W. Magnus and S. Winkler. Hill's Equation. Interscience Publishers (Wiley), New York, 1966.

[11] Mauro Mobilia. Oscillatory dynamics in rock-paper-scissor games with mutations. Journal of Theoretical Biology, 2010.

[12] A. H Nayfeh and D. T. Mook. Nonlinear Oscillations. Wiley, NY, 1979.

[13] Martin A. Nowak. Evolutionary Dynamics: Exploring the Equations of Life. The Belknap Press of Harvard University Press, 2006. 
[14] Richard H. Rand. Lecture notes on nonlinear vibrations (version 53), May 2012.

[15] Richard H. Rand, Max Yazhbin, and David G. Rand. Evolutionary dynamics of a system with periodic coefficients. Communications in Nonlinear Science and Numerical Simulation, 2011.

[16] Geoff Recktenwald and Richard H. Rand. Coexistence phenomenon in autoparametric excitation of two degree freedom systems. International J. Nonlinear Mechanics, 2005.

[17] Dirk Semmann, Hans-Jurgen Krambeck, and Manfred Milinski. Volunteering leads to rock-paper-scissors dynamics in a public goods game. Nature, 2003.

[18] B. Sinervo and C. M. Livelyi. The rock-paper-scissors game and the evolution of alternative male strategies. Nature, 1996.

[19] J. Maynard Smith and G.R. Price. The logic of animal conflict. Nature, 1973.

[20] James J. Stoker. Nonlinear Vibration in Mechanical and Electrical Systems. Wiley, NY, 1950.

[21] Steven H. Strogatz. Nonlinear Dynamics and Chaos: With Applications to Physics, Biology, Chemistry, and Engineering. Westview Press, Cambridge, MA, 1994.

[22] T.L. Vincent and J.S. Brown. Evolutionary Game Theory, Natural Selection, and Darwinian Dynamics. Cambridge University Press, 2005.

[23] John von Neumann and Oskar Morgenstern. Theory of Games and Economic Behavior. Princeton University Press, 1944. 